\title{
PALYNOLOGY OF EL FOYEL GROUP (PALEOGENE), ÑIRIHUAU BASIN, ARGENTINA
}

\author{
MIRTA E. QUATTROCCHIO, MARCELO A. MARTÍNEZ \\ Departamento de Geología, Instituto Geológico del Sur, Universidad Nacional del Sur, CONICET, San Juan 670, 8000, \\ Bahía Blanca, Argentina.mquattro@criba.edu.ar,martinez@criba.edu.ar \\ MARCOS A. ASENSIO \\ TECPETROL, Bahía Blanca, Argentina.marcos.asensio@tecpetrol.com \\ M. ELINA CORNOU \& DANIELA E. OLIVERA \\ Departamento de Geología, Universidad Nacional del Sur, San Juan 670, 8000, Bahía Blanca, Argentina. \\ elina.cornou@uns.edu.ar,danielaolivera@yahoo.com.ar
}

\begin{abstract}
This work aims to analyze the paleovegetation, paleoclimates and paleoceanography of the Paleogene of the Río Foyel section (El Foyel Group), Ñirihuau Basin, Argentina. It comprises Troncoso, Salto del Macho and Río Foyel formations. Sporomorph analysis reflects a regional forest dominated by Nothofagaceae, Myrtaceae, Podocarpaceae and Palmae, developed under a temperate to warm-temperate and humid climate. In the studied section, the relation between terrestrial/marine palynomorphs is considered. In the Troncoso Formation, terrigenous palynomorphs dominate over marine elements. The recurrent presence of gymnosperm pollen with Araucariaceae indicates temperate and humid conditions. The Salto del Macho Formation shows a retraction of the gymnosperm forest, associated with the dominance of Nothofagus forest, indicating a temperate and humid condition. In the Río Foyel Formation, the sporomorphs indicate the presence of a temperate to warm-humid forest with subordinate open areas and a remarkable input from the coastal environment. In the same formation, the palynological characteristics, which indicate upward shallowing, allow the identification of parasequences. The bounding surfaces of parasequences are defined as surfaces of flooding which represent a relative sea level rise (marine flooding surfaces). The Río Foyel Formation shows an upward shallowing sequence, indicated by the decrease in abundance and diversity of dinocysts. Different marine flooding surfaces were detected, associated with the diversity and abundance of dinocyst. A comparison of these spore-pollen assemblages with others from Patagonia, using multivariate statistic techniques, yields strong similarities between the Troncoso Formation and the Sloggett Formation (late Eocene to ?early Oligocene); and between Salto del Macho and Río Foyel formations with Loreto Formation (late Eocene-Oligocene) and San Julián Formation in cabo Curioso area (Oligocene).
\end{abstract}

Key words: Paleogene, Palynology, El Foyel Group, Ñirihuau Basin, Argentina.

RESUMO - Este trabalho visa analisar a paleovegetação, os paleoclimas e a paleoceanografia do Paleógeno da região do Río Foyel (Grupo Foyel), bacia Nirihuau, Argentina, compreendendo as formações Troncoso, Salto del Macho e Rio Foyel. A análise de esporomorfos mostra a existência de um bosque regional formado principalmente por Nothofagaceae, Myrtaceae, Podocarpaceae e Palmae, desenvolvido sob clima temperado a temperado-quente e úmido. Na região estudada, foi considerada a relação entre os palinomorfos continentais/marinhos. Há predominância de palinomorfos terrígenos sobre elementos marinhos na Formação Troncoso. A recorrência de pólen de gimnospermas como Araucariaceae indica clima temperado a úmido. A Formação Salto del Macho mostra uma regressão do bosque de gimnospermas, relacionada ao avanço do bosque de Nothofagus, sugerindo clima temperado-úmido. Na Formação Río Foyel, os esporomorfos demonstram a presença de um bosque temperado a quente-úmido, com áreas abertas subordinadas e um notável input do ambiente costeiro. Na mesma formação, as características palinológicas, que indicam rasamento ascendente, permitem a identificação de parasequências. Os limites das parasequências são definidos como superfícies de inundação, as quais representam um aumento relativo do nível do mar (superfícies de inundação marinha). A Formação Río Foyel mostra uma sequência de rasamento ascendente, indicada pela diminuição de dinocistos. Foram detectadas diferentes superfícies de inundação marinha, associadas à diversidade e abundância de dinocistos. A comparação entre estas associações esporopolínicas com outras da Patagônia, realizada através de métodos estatísticos multivariados, revela uma grande semelhança entre a Formação Troncoso (Eoceno médio a superior) e a Formação Slogett (Eoceno superior a ?Oligoceno inferior); e entre as formações Salto del Macho e Río Foyel com associações da Formação Loreto (Eoceno superior-Oligoceno) e a Formação San Julián na área do cabo Curioso (Oligoceno).

Palavras-chave: Paleógeno, Palinologia, Grupo El Foyel, bacia Nirihuau, Argentina. 


\section{INTRODUCTION}

This paper focuses on the application of palynological studies to determine relationships between palynomorph concentrations and associations of the biosphere to reconstruct plant associations, oceanic conditions and to interpret climatic trends.

Its goal is to contribute to understand the paleovegetation, paleoclimates and paleoceanographic conditions of El Foyel Group (Paleogene) at the Río Foyel type section, in the Nirihuau Basin. The Troncoso, Salto del Macho and Río Foyel formations of El Foyel Group are here included.

The major vegetation types may be recognized from the pollen assemblage. Many Tertiary pollen types show affinities with living taxa, hence it should be possible to use the ecological tolerance of today to reconstruct Tertiary climates; though not always satisfactorily due to the fact that ecological tolerances may have changed over the time as suggested by Martin (1987, 1997). Among the most important proxies that were used to detect paleoenvironmental changes are the organic-walled dinoflagellate cysts (dinocysts). Dinocyst assemblages in sediments provide information pertinent to the ecology of the water in which the dinoflagellates lived.

\section{PREVIOUS INVESTIGATIONS ON PALEONTOLOGY}

The Río Foyel Formation contains an abundant fauna of marine invertebrates, which have been studied since early 20th century (von Ihering, 1904, 1907, 1914; Gonzalez Bonorino, 1944). In samples from the same section studied here, but from the upper levels, Bertels (1980, 1993, 1994a,b) found strong similarities between the assemblages of foraminifera and those from the Monte León Formation (Santa Cruz Province, Atlantic coast). Based in this bio-correlation, Bertels (1980, 1993, 1994a, b) postulated an Oligocene age for the Río Foyel Formation. Recently, Malumián et al. (2008) found moulds of Transversigerina $\mathrm{cf}$. T. transversa (Cushman) at $18 \mathrm{~m}$ from the base of the section under study. This genus appeared in the late Oligocene and the compared species in the early Miocene. Chiesa \& Camacho (2001), based on the megafauna, proposed a middle Eocene age for the lower part of this unit.

The megaflora collected by Washburne between 1911 and 1912 in the Nirihuau Formation was the basis of Berry's (1928) studies about South American flora. Romero \& Dibbern (1984) provide a detailed report on the megaflora in the studied area.

Previous palynological studies correspond to the Huitrera, Salto del Macho and Río Foyel formations (Melendi et al., 2003; Barreda et al., 2003; Martínez et al., 2008) and in the "Serie Andesítica" (Sepúlveda, 1980). The Huitrera Formation (Melendi et al., 2003) presents microfloras without Nothofagidites of the early Eocene and microfloras with Nothofagidites ("brassi" and "fusca" groups) of the middle to late Eocene. Based on the fungal assemblage, Sepúlveda (1980) associated the "Serie Andesítica", in the Cordón
Oriental del Futalaufquen, to the middle Eocene. Other samples from the upper levels of this same section (not studied here) (Barreda et al., 2003) recognize a similar palynological assemblages, but less diverse and they postulated a late Oligocene to early Miocene age taking into account the presence of Tuberculodinium vancampoae (Rossignol) Wall. However, this species has already been accurately recognized as corresponding to the early Oligocene (see Head et al., 1989, p. 440). Martínez et al. (2008) provide the sedimentology, environmental conditions and an introduction of the plant communities of the Salto del Macho Formation.

\section{STRATIGRAPHY}

The fill of the Tertiary Nirihuau Basin, which is located on the eastern Subandean flank of the Northern Patagonian Cordillera, is characterized by two major volcanic and sedimentary cycles. The Base Cycle (late Paleocene to early Oligocene) involves the Pilcaniyeu Volcanic Belt (Huitrera Formation) and the Cerro Bastión, Río Foyel ("Patagonian") and Rincón de Cholila formations. The Top Cycle (early Oligocene to Pliocene) is represented by the Nahuel Huapi Group and consists, from base to top, of the El Maitén Volcanic Belt (Ventana Formation) and the Norquinco, Ñirihuau and Collón Cura formations (Asensio et al., 2010). Structurally, the western part of the basin has taken part in the deformation process that gave rise to the Nirihuau fold and thrust belt, whereas the eastern border accounts for the basin platform showing poor or no-deformation, with three distinct regions: North (Ñirihuau), South (Ñorquinco) and West (El Bolsón).

El Foyel Group (Asensio et al., 2005) is stratigraphically located between the vulcanite of the Cerro Bastion Formation (middle Eocene, Sepúlveda, 1980) and the vulcanite of the Ventana Formation (late Oligocene, Cazau et al., 1989). It reaches a thickness of more than $1900 \mathrm{~m}$. It appears as if El Foyel Group has a chronostratigraphic range extending from the middle Eocene to the early Oligocene. This entity presents a variable vertical development, as evidenced by the margins of the Foyel River, where the succession reaches a thickness of $2000 \mathrm{~m}$ (Asensio et al., 2005).

\section{Río Foyel Section}

The outcropping section of the Foyel river margins $\left(42^{\circ} \mathrm{S}\right)$ (Figure 1) is central to the stratigraphic analysis of the Nirihuau Basin. Although it has been known since the 19th century (Roth, 1899; Wherli, 1899), only in 2005 it was substantially studied and valued as having important implications within the sedimentary register of the basin. From the bottom up, it comprises Troncoso, Salto del Macho and Río Foyel formations (Figure 2).

The Troncoso Formation (Asensio et al., 2005) reaches a thickness of $750 \mathrm{~m}$ and is constituted mainly of volcaniclastic sandstones and tobaceous clay rocks with intercalation of thick ignimbritic banks. A particularity of this unity is that it presents a divergence between its constitutive layers, which 

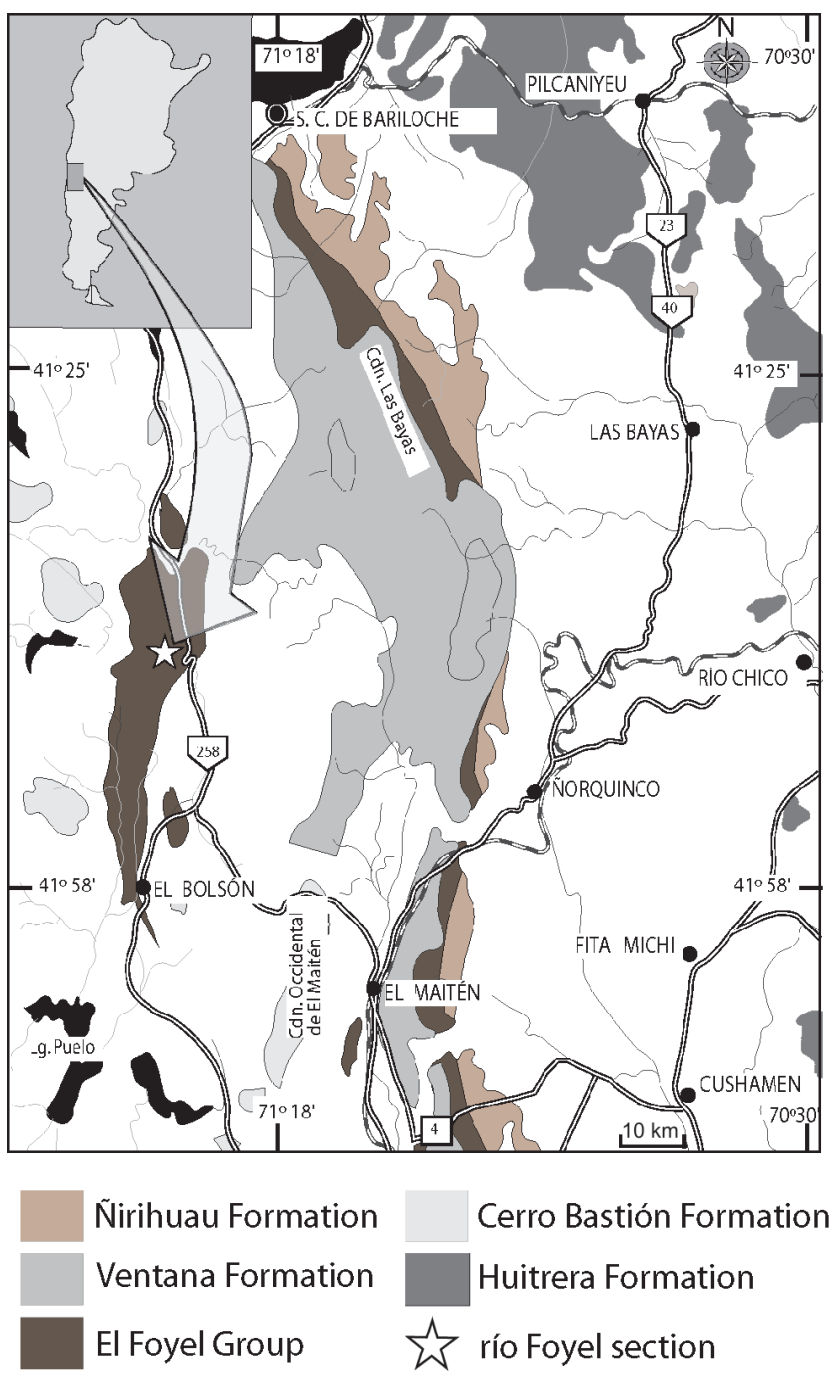

Figure 1. Location map of the study area with indication of the Río Foyel section.

can be inferred as resulting from synsedimentary tectonic activity. The contact with the Cerro Bastion Formation is sharp, although in most cases it is blurred by heavily forested areas. The age of this unit has not been established with precision but, as inferred from its stratigraphic relations, it is estimated to extend from the middle Eocene to late Eocene (Asensio et al., 2005).

The Salto del Macho Formation reaches $560 \mathrm{~m}$ of thickness and is characterized by having tabular bodies-less than $10 \mathrm{~m}$ thick- with a predominance of the conglomeradic facies, among which there is intercalation of sandstone banks and, in lower proportion, carbonaceous shale. As evidenced by the comparison of facies and the stratigraphic relations within the section, it is possible to emphasize that the origin of this dense succession, mainly conglomeradic, is closely related to subaerial hyperconcentrated flows, associated to distal deltaic fans. The tectosedimentary development of the unit is linked with a strong extensional pulse occurred during middle Eocene-lower Oligocene and is related with the tectonics of the Farallón and the Sudamericana Plates (Asensio et al., 2008). The age of this unit as inferred from

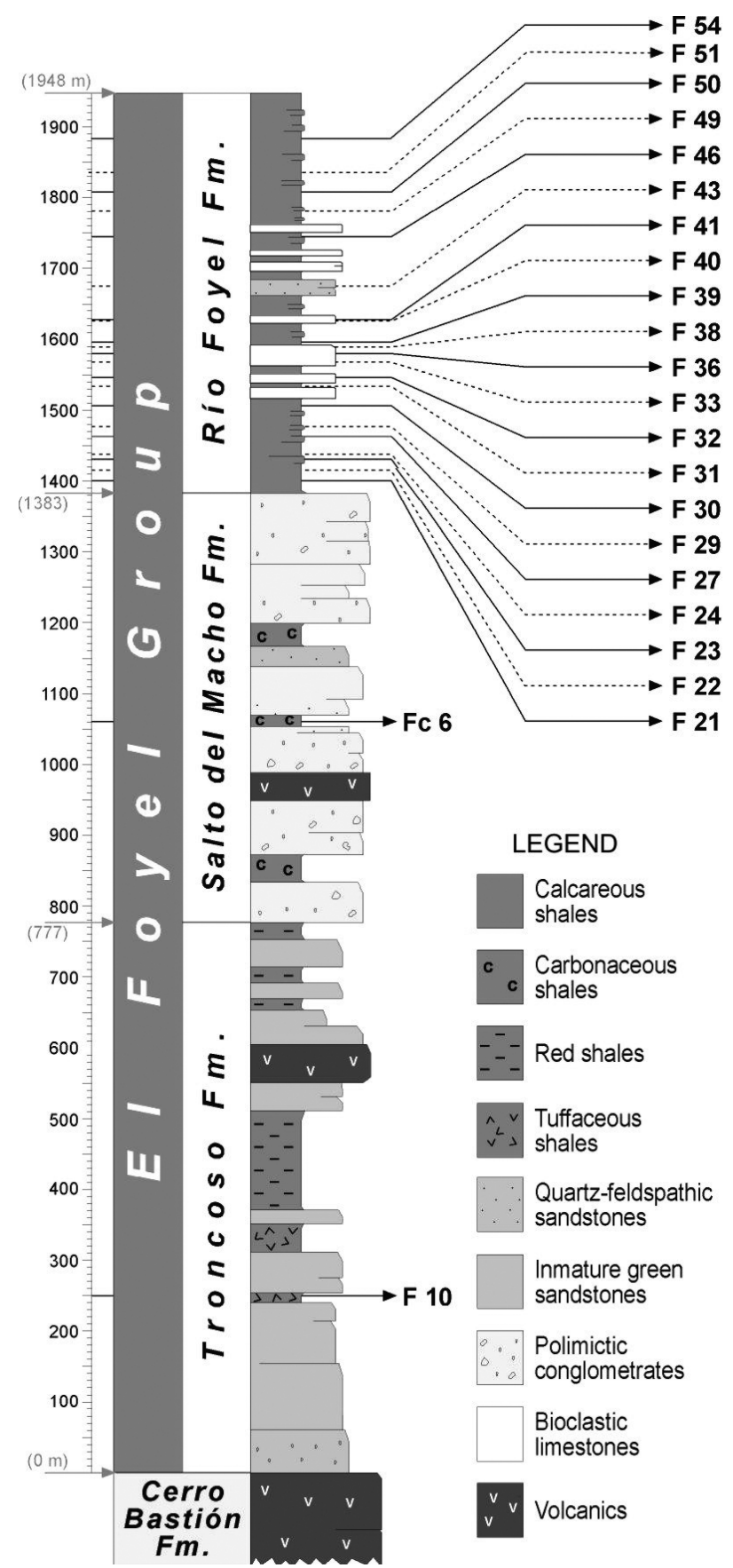

Figure 2. Study section and stratigraphic distribution of the palynological samples.

its stratigraphic relations extends from the middle Eocene to early Oligocene (Asensio et al., 2005).

The Río Foyel Formation (Pöthe de Baldis, 1984) presents a minimum thickness higher than $600 \mathrm{~m}$ and is constituted mainly by black calcareous shales. The age is determined by a sampling ${ }^{87} \mathrm{Sr} /{ }^{86} \mathrm{Sr}$ of $30,65 \mathrm{Ma}$ (Griffin in Asensio et al., 2010) on the valve of an Ostrea and on an andesite dyke that intrudes to the unit and which yielded the value of 31 $\mathrm{Ma}(\mathrm{K} / \mathrm{Ar})$ (Giacosa et al., 2001). It stands, with a maximum flooding surface, on the conglomerates of the Salto del Macho Formation and could be related to the sandstones of the Norquinco Formation (Cazau, 1972). 


\section{MATERIAL AND METHODS}

Physical and chemical extraction was performed using standard palynological processing techniques (Volkheimer $\&$ Melendi, 1976). Twenty-three samples were processed for palynological purpose, and sixteen yielded moderately to poorly preserved palynomorphs. Six from Troncoso Formation only one fertile, one from Salto del Macho Formation and the others from Río Foyel Formation. All figured specimens are lodged in the collections of the Palynological Laboratory (UNSP), Universidad Nacional del Sur, Bahía Blanca, Argentina. In the citation of specimens referred to or illustrated, the sample and slide number (e.g. 1980a) are given first and the position on the slide is indicated by an England Finder reference (e.g. G36/4). Additionally, the formations are given: Salto del Macho (SM), Troncoso (T), Río Foyel (RF).

The palynologic analysis for biostratigraphic (palynostratigraphic), paleoclimatical and paleoecological approach is based on qualitative and quantitative data. The quantitative analysis comprises a minimum of 100 palynomorphs per sample, excepting those samples in which this was not possible due the scarcity of palynomorphs. These are indicated in Table 1 as "presence". The sporomorphs are arranged in Table 2 according to their botanical affinity.

Hammer's et al. (2001) program, Palaeontological Statistics (PAST), was used to determine similarities among El Foyel Group and other Danian-Miocene sporomorph assemblages from different units of southern South America, based on presence/absence data.

Each site in the cluster represents a sum of several samples with the exception of Troncoso, Salto del Macho (El Foyel Group) and San Julián formations in cabo Curioso (Santa Cruz Province). Using PAST, the Jaccard Coefficient was applied as a measure of similarity (between 0 to 1 - low to high similarity) between samples. The results of the cluster analyses are presented in dendrograms (Figure 3).

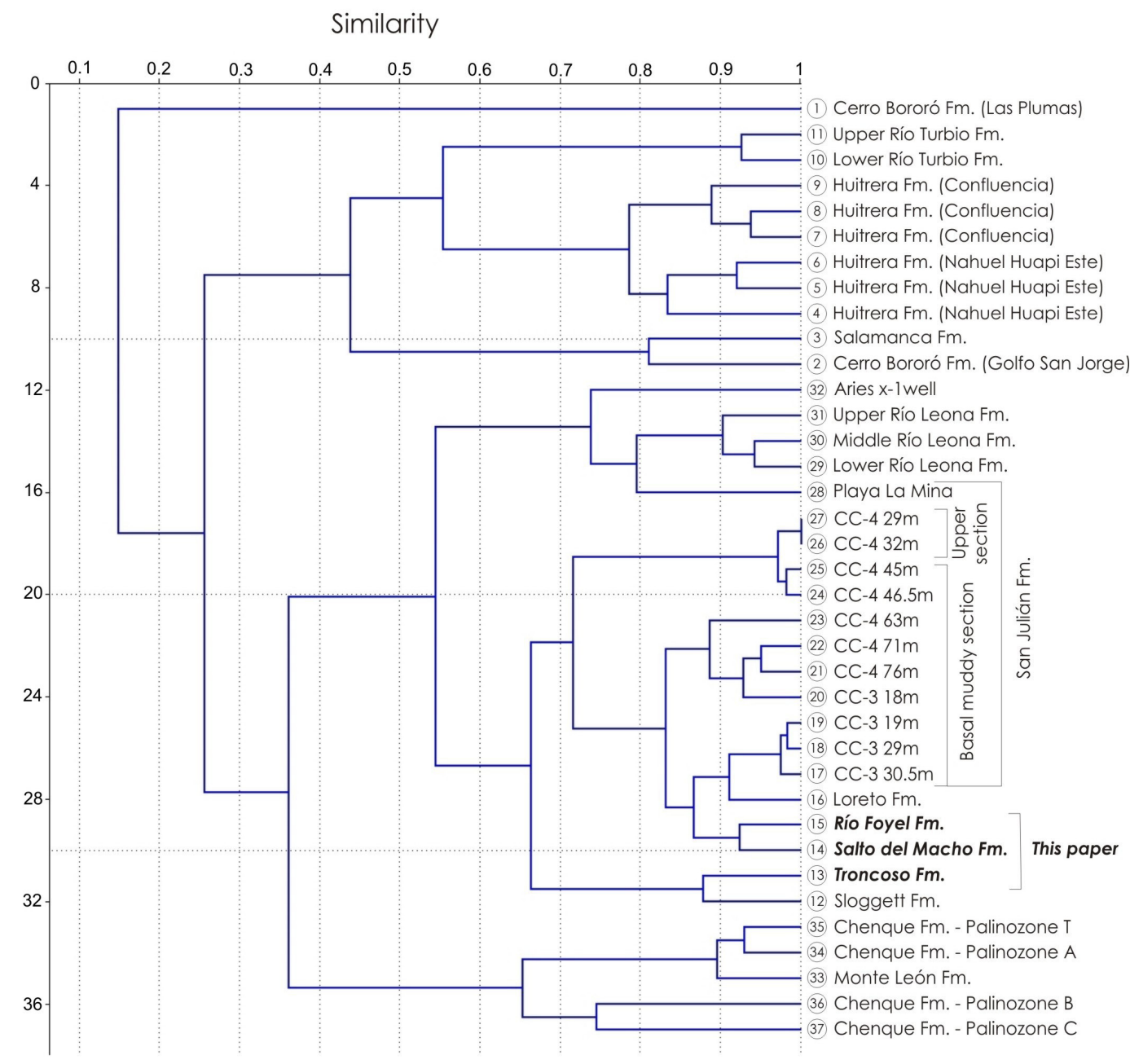

Figure 3. Dendrogram showing the clustering of palynological assemblages from different formations and/or localities, based on Jaccard coefficient and the PAST programme. References: 1, Volkheimer et al. (2007); 2-3, Archangelsky (1973, 1976); Archangelsky \& Romero (1974); Archangelsky \& Zamaloa (1986); 4-9, Melendi et al. (2003); 10-11, following in this paper the Lower and Upper Section (Rio Turbio Formation) recognized by Hünicken (1955); Archangelsky (1972); Romero \& Castro (1986); Romero \& Zamaloa, 1985; Romero, 1977; 12, Olivero et al., 1998; 13-15, Asensio et al., 2005; this paper; 16, Fasola (1969); 17-27, Nañez et al. (2009); 28, Barreda (1997); 29-31, Barreda et al. (2009); 32, Palamarczuk \& Barreda (2000); 33, Barreda \& Palamarczuk (2000a); 34-37, Barreda (1996); Palamarczuk \& Barreda (1998); Barreda \& Palamarczuk (2000b) (modified from Nañez et al., 2009). 
Table 1. Distribution charts of palynomorphs from the río Foyel section.

\begin{tabular}{|c|c|c|c|c|c|c|c|c|c|c|c|c|c|c|c|c|c|}
\hline \multirow{2}{*}{ Taxa } & Formation / Samples & $\begin{array}{l}\overrightarrow{0} \\
0 \\
0 \\
0 \\
0 \\
0\end{array}$ & 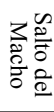 & \multicolumn{14}{|c|}{ Río Foyel } \\
\hline & & તิ & ลิ & নे & สั & 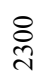 & तิ & $\underset{\sim}{\sim}$ & సे & ָి & $\overline{\widetilde{\Omega}}$ & 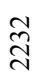 & $\stackrel{\widetilde{N}}{\overparen{N}}$ & $\stackrel{\circ}{\curvearrowright}$ & 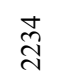 & $\bar{\nabla}$ & $\frac{\sim}{\curvearrowright}$ \\
\hline 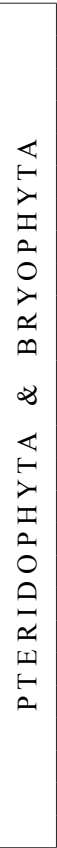 & $\begin{array}{l}\text { Azolla } \text { sp. } \\
\text { Baculatisporites comaumensis } \\
\text { Baculatisporites turbioensis } \\
\text { Baculatisporites sp. } \\
\text { Biretisporites crassilabratus } \\
\text { Biretisporites sp. } 1 \\
\text { Cingutriletes australis } \\
\text { Concavisporites sp. } \\
\text { Cyatheacidites annulatus } \\
\text { Cyathidites subtilis } \\
\text { Deltoidospora australis } \\
\text { Deltoidospora minor } \\
\text { Deltoidospora spp. } \\
\text { Dictyophyllidites harrisii } \\
\text { Gleicheniidites argentinus } \\
\text { Hymenophylleaceae } \\
\text { cf. Hymenophyllum caudiculatum } \\
\text { Ischyosporites areapunctatis } \\
\text { Laevigatosporites ovatus } \\
\text { Leptolepidites macroverrucosus } \\
\text { Matonisporites ornamentalis } \\
\text { Muricingulisporis chenquensis } \\
\text { Peromonolites vellosus } \\
\text { Polypodiisporites inangahuensis } \\
\text { Reticuloidosporites tenellis } \\
\text { Indeterminate trilete spore }\end{array}$ & $\begin{array}{l}0.9 \\
0.9\end{array}$ & $\begin{array}{c}3.3 \\
3.3 \\
X\end{array}$ & $\begin{array}{l}\mathrm{X} \\
\mathrm{X}\end{array}$ & $\mathrm{X}$ & $\mathrm{X}$ & $\begin{array}{l}10.6 \\
9.4 \\
4.7\end{array}$ & $\begin{array}{l}4.9 \\
4.9\end{array}$ & $\begin{array}{l}X \\
X \\
X\end{array}$ & $\begin{array}{l}20.9 \\
11.6\end{array}$ & $\begin{array}{l}2.3 \\
2.3 \\
2.3 \\
2.3\end{array}$ & $\begin{array}{l}X \\
X\end{array}$ & $\begin{array}{l}8.8 \\
6.6\end{array}$ & $\begin{array}{l}57.3 \\
13.3\end{array}$ & $\begin{array}{l}1.2 \\
9.8\end{array}$ & $\begin{array}{l}12.3 \\
1.4 \\
2.7\end{array}$ & $\begin{array}{l}3.2 \\
3.2\end{array}$ \\
\hline 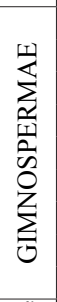 & $\begin{array}{l}\text { Araucariacites australis } \\
\text { ? Dacrycarpites australis } \\
\text { Inaperturopollenites spp. } \\
\text { Microcachryidites antarcticus } \\
\text { Phyllocladidites mawsonii } \\
\text { Podocarpidites marwickii } \\
\text { Podocarpidites spp. } \\
\text { Taxodiaceaepollenites hiatus } \\
\text { Trisaccites microsaccatum }\end{array}$ & $\begin{array}{c}33.6 \\
1.8 \\
\\
10.6 \\
0.9\end{array}$ & $\begin{array}{l}1.7 \\
1.7 \\
6.7\end{array}$ & $\mathrm{X}$ & $\mathrm{X}$ & $\begin{array}{l}X \\
X\end{array}$ & $\begin{array}{c}11.8 \\
5.9 \\
2.4\end{array}$ & $\begin{array}{c}11 \\
4.9 \\
3.7\end{array}$ & $\mathrm{X}$ & 2.3 & $\begin{array}{c}11.6 \\
4.6 \\
\\
14\end{array}$ & $\mathrm{X}$ & $\begin{array}{l}3.3 \\
2.2\end{array}$ & 1.3 & $\begin{array}{c}1.2 \\
6.1 \\
11 \\
4.8\end{array}$ & $\begin{array}{l}13.7 \\
13.7\end{array}$ & 1.6 \\
\hline$\frac{\mathscr{G}}{\mathbb{E}}$ & $\begin{array}{l}\text { Botryococcus } \mathrm{sp} . \\
\text { ? Celyphus rallus }\end{array}$ & 11.5 & & & & & & 1.2 & & & & & & & 1.2 & & \\
\hline 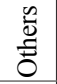 & $\begin{array}{l}\text { Foraminiferal test linings } \\
\text { ? Partitomorphitae }\end{array}$ & & & & & & & 1.2 & & & & & & 1.3 & 4.9 & 1.4 & \\
\hline $\begin{array}{l}\text { I } \\
\varangle \\
\Sigma \\
\Sigma \\
\simeq \\
\text { 工1 } \\
a \\
\text { n } \\
0 \\
- \\
0 \\
z \\
\varangle\end{array}$ & $\begin{array}{l}\text { Baumannipollis sp. } \\
\text { Chenopodipollis chenopodiaceoides } \\
\text { Corsinipollenites atlantica } \\
\text { Graminidites sp. } \\
\text { Haloragacidites } \mathrm{sp} . \\
\text { Myrtaceidites verrucosus } \\
\text { Myrtaceidites sp. (cf. Myrceugenia) } \\
\text { Momipites sp. } \\
\text { Nothofagidites americanus } \\
\text { Nothofagidites anisoechinatus } \\
\text { Nothofagidites dorotensis } \\
\text { Nothofagidites flemingii } \\
\text { Nothofagidites menziesii type } \\
\text { Nothofagidites nanus } \\
\text { Nothofagidites rocaensis } \\
\text { Nothofagidites saraensis } \\
\text { Nothofagidites spp. } \\
\text { Propylipollis rynthius } \\
\text { Proteacidites subscabratus } \\
\text { Proteacidites symphyonemoides } \\
\text { Proteacidites spp. } \\
\text { Pseudowinterapollis couperi } \\
\text { Psilamonocolpites grandis } \\
\text { Psilatricolporites salamanquensis } \\
\text { Psilatricolporites spp. } \\
\text { Restioniidites sp. }\end{array}$ & 0.9 & $\begin{array}{c}11.7 \\
5 \\
11.7\end{array}$ & X & $\mathrm{X}$ & $\begin{array}{l}X \\
X \\
X \\
X \\
X\end{array}$ & $\begin{array}{l}8.2 \\
3.5 \\
2.4\end{array}$ & $\begin{array}{l}4.9 \\
4.9 \\
1.2\end{array}$ & $\begin{array}{l}X \\
X \\
X\end{array}$ & $\begin{array}{l}2.3 \\
2.3\end{array}$ & 2.3 & $\begin{array}{l}X \\
X\end{array}$ & $\begin{array}{l}4.4 \\
3.3\end{array}$ & 1.3 & $\begin{array}{c}11 \\
3.7\end{array}$ & $\begin{array}{l}1.4 \\
4.1 \\
1.4\end{array}$ & $\begin{array}{r}4.8 \\
11.1\end{array}$ \\
\hline
\end{tabular}


Table 1. Cont.

\begin{tabular}{|c|c|c|c|c|c|c|c|c|c|c|c|c|c|c|c|c|c|}
\hline \multirow{2}{*}{\multicolumn{2}{|c|}{ Taxa Formation / Samples }} & $\begin{array}{l}\overrightarrow{0} \\
\overrightarrow{0}\end{array}$ & 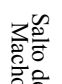 & \multicolumn{14}{|c|}{ Río Foyel } \\
\hline & & $\stackrel{\widetilde{\Xi}}{\tilde{N}}$ & ते & ते & $\begin{array}{l}\text { ते } \\
\text { ते }\end{array}$ & ষ্ণ & $\widehat{\widehat{N}}$ & $\stackrel{\infty}{\mathbb{N}}$ & तิ & $\begin{array}{l}\stackrel{N}{N} \\
\text { त. }\end{array}$ & $\overline{\tilde{N}}$ & तี & 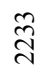 & 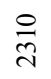 & त્ત & $\vec{\nabla}$ & $\frac{\sim}{\vec{n}}$ \\
\hline 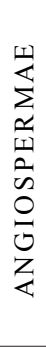 & $\begin{array}{l}\text { Rhoipites baculatus } \\
\text { Rhoipites minusculus } \\
\text { Rhoipites sp. A } \\
\text { Rhoipites spp. } \\
\text { cf. Rousea patagonica } \\
\text { Spinizonocolpites sp. } \\
\text { Striatricolporites spp. } \\
\text { Triatriopollenites bertelsii } \\
\text { Triorites minor } \\
\text { Indet. tricolporate pollen grain }\end{array}$ & $\begin{array}{c}0.9 \\
2.65 \\
2.65\end{array}$ & 1.7 & & & $X$ & 3.5 & 1.2 & $\mathrm{X}$ & 2.3 & 4.6 & $X$ & & 1.3 & $\begin{array}{l}2.4 \\
1.2\end{array}$ & $\begin{array}{l}1.4 \\
4.1\end{array}$ & 1.6 \\
\hline $\begin{array}{l}\vec{U} \\
Z \\
\supset \\
\supset \\
\end{array}$ & $\begin{array}{l}\text { Desmidiospora willoughbyi } \\
\text { cf. Diporicellaesporites navicularis } \\
\text { Diporisporites } \mathrm{cf} \text {. psilatus } \\
\text { Fractisporonites } \mathrm{sp} \text {. } \\
\text { Hilidicellites } \mathrm{sp} \text {. } \\
\text { Hypoxylonites } \mathrm{sp} . \\
\text { Inapertisporites cystoides } \\
\text { Inapertisporites elencantense } \\
\text { Monoporites abruptus } \\
\text { Monoporisporites lagenarius } \\
\text { Multicellaesporites } \mathrm{sp} . \\
\text { Multicellites } \mathrm{sp} .\end{array}$ & $\begin{array}{l}X \\
X\end{array}$ & & & & $X$ & $\mathrm{X}$ & & $\begin{array}{l}X \\
X\end{array}$ & $\mathrm{X}$ & & & $\mathrm{X}$ & & $X$ & & $\mathrm{X}$ \\
\hline $\begin{array}{l}n \\
1 \\
0 \\
\lambda \\
0 \\
0 \\
Z \\
\square \\
0\end{array}$ & $\begin{array}{l}\text { ? Apteodinium sp. } \\
\text { ? Batiacasphaera baculata } \\
\text { Batiacasphaera micropapillata } \\
\text { Batiacasphaera } \text { spp. } \\
\text { ? Cristadinium sp. } \\
\text { Escharisphaeridia psilata } \\
\text { Escharisphaeridia spp. } \\
\text { Hystrichostrogylon sp. } \\
\text { Lejeunecysta communis } \\
\text { Lejeunecysta convexa } \\
\text { Lejeunecysta fallax } \\
\text { Lejeunecysta globosa } \\
\text { Lejeunecysta spp. } \\
\text { Lingulodinium bergmannii } \\
\text { Lingulodinium hemicystum } \\
\text { cf. Selenopemphix nephroides } \\
\text { Spiniferites } \text { sp. } \\
\text { Tuberculodinium vancampoae } \\
\text { Proximate indet. dinocysts }\end{array}$ & $\begin{array}{l}2.7 \\
2.7 \\
0.9\end{array}$ & 3.3 & $\mathrm{X}$ & $X$ & $\begin{array}{l}X \\
X\end{array}$ & $\begin{array}{l}2.4 \\
X\end{array}$ & 1.2 & $X$ & 4.7 & $\begin{array}{c}2.3 \\
7\end{array}$ & $\mathrm{X}$ & $\begin{array}{l}5.5 \\
9.9 \\
6.6\end{array}$ & 2.7 & 1.2 & $\begin{array}{c}12.3 \\
1.4 \\
1.4\end{array}$ & $\begin{array}{l}1.6 \\
6.4\end{array}$ \\
\hline
\end{tabular}

The "non-metric multidimensional scaling" (N-MDS) (Kruskal \& Wish, 1976) was used as well, which is based on rank-order of the elements of the similarity matrix, rather than on their absolute values. The procedure is based on a clear assumption: the greater the similarity between two objects, the closer to each other they will appear in the ordination (Kovach, 1989). The results presented in here correspond to the first two axes, which reflect the main variability of the data (Figure 4).

\section{PALEOCOMMUNITIES AND PALEOCLIMATIC INFERENCES}

Sporomorphs analysis (Figures 5-7) allows the characterization of the paleofloristic and paleoclimatic scenario of the studied section during the middle Eocene-Oligocene. It reflects a regional forest dominated by Nothofagaceae, Myrtaceae, Podocarpaceae and Palmae, developed under a temperate to warm-temperate and humid climate. The distribution charts of palynomorphs and the list of identified species, and botanical affinities from the río Foyel section are given in Tables 1 and 2 , respectively.

In río Foyel section, the species of Nothofagidites correspond to N. saraensis Menéndez \& Caccavari and N. rocaensis Romero, which are both similar to the Nothofagus "Dombeyi type" and, among others, include the actual $N$. dombeyi (Mirb.) Oerst and N. antarctica (Forst.) Oerst. These two species are opposite ecologic indicators. The first corresponds to the evergreen forest and the second to the microtermic and deciduous forest. The paleoenviromental inferences with these morpho-species then are limited.

Morphologically different pollen types are recognizable within the genus Nothofagus Blume, but a single pollen type can be found in a number of living species (Dettmann \& Pocknall, 1990). This point is important, for the ecological tolerances apply to the species and if the identification is to more than one species, their ecological tolerances may not be the same (Martin, 1997). 
Table 2. List of identified species, and botanical affinities.

\begin{tabular}{|c|c|}
\hline Fossil taxon & Botanical affinity \\
\hline $\begin{array}{l}\text { Bryophyte and Pteridophyte spores } \\
\text { Azolla sp. } \\
\text { Baculatisporites comaumensis (Cookson) Potonié, } 1953 \\
\text { Baculatisporites turbioensis Archabgelsky, 1972 } \\
\text { Baculatisporites sp. (in Barreda et al., 2003) } \\
\text { Biretisporites crassilabratus Archangelsky, } 1972 \\
\text { Biretisporites sp. } 1 \text { (in Archangelsky, 1972) } \\
\text { Cingutriletes australis (Cookson) Archangelsky, } 1972 \\
\text { Concavisporites sp. } \\
\text { Cyatheacidites annulatus Cookson, } 1947 \\
\text { Cyathidites subtilis Partridge, 1976 } \\
\text { Deltoidospora australis (Couper) Pocock, } 1970 \\
\text { Deltoidospora minor (Couper) Pocock, } 1970 \\
\text { Dictyophyllidites harrisii Couper, 1958 } \\
\text { Gleicheniidites argentinus Volkheimer, } 1972 \\
\text { Hymenophylleaceae } \\
\text { cf. Hymenophyllum caudiculatum Mart. } \\
\text { Ischyosporites aerapunctatis (Stuchlik) Barreda, } 1996 \\
\text { Laevigatosporites ovatus Wilson \& Webster, } 1946 \\
\text { Leptolepidites macroverrucosus Schulz, } 1967 \\
\text { Matonisporites ornamentalis (Cookson) Partridge in Stover \& Partrridge, } 1973 \\
\text { Muricingulisporis chenquensis Barreda, } 1992 \\
\text { Peromonolites vellosus Partridge, } 1973 \\
\text { Polypodiisporites inangahuensis (Couper) Potonié, } 1956 \\
\text { emend. Pocknall \& Mildenhall, } 1984 \\
\text { Reticuloidosporites tenellis Krutzsch, } 1959\end{array}$ & $\begin{array}{c}\text { ?Hymenophylleaeceae } \\
\text { Sphagnales (Sphagnum) } \\
\text { ?Filicopsida } \\
\text { Lophosoriaceae (Lophosoria) } \\
\text { Cyatheaceae/Dicksoniaceae } \\
\text { Polypodiaceae (Acrostichum) } \\
\text { Polypodiaceae (Acrostichum) } \\
\text { Matoniaceae (Phlebopteris) } \\
\text { Gleicheniaceae } \\
\text { Hymenophylleaeceae } \\
\text { Hymenophylleaeceae } \\
\text { Dicksoniaceae } \\
\text { Blechnaceae } \\
\text { Dennstaedtiaceae } \\
\text { Dicksoniaceae } \\
\text { Pteridaceae (Pteris semiadnata) } \\
\text { Blechnaceae } \\
\text { Polypodiaceae } \\
\text { Polypodiaceae }\end{array}$ \\
\hline $\begin{array}{l}\text { Gimnosperm pollen } \\
\text { Araucariacites australis Cookson, } 1947 \\
\text { ? Dacrycarpites australiensis Cookson \& Pike, } 1953 \\
\text { Microcachryidites antarcticus Cookson } 1947 \text { ex Couper, } 1953 \\
\text { Phyllocladidites mawsonii Cookson, } 1947 \\
\text { Podocarpidites marwickii Couper, } 1953 \\
\text { Taxodiaceaepollenites hiatus (Potonie) Kremp } \\
\text { Trisaccites microsaccatum (Cookson) Couper, } 1960\end{array}$ & $\begin{array}{c}\text { Araucariaceae (Araucaria) } \\
\text { Podocarpaceae } \\
\text { Podocarpaceae (Microcachrys) } \\
\text { Podocarpaceae (Dacrydium franklinii) } \\
\text { Podocarpaceae } \\
\text { Taxodiaceae } \\
\text { Podocarpaceae }\end{array}$ \\
\hline $\begin{array}{l}\text { Angiosperm pollen } \\
\text { Baumannipollis sp. } \\
\text { Chenopodipollis chenopodiaceoides (Martin) Truswell, } 1985 \\
\text { Corsinipollenites atlantica Barreda, } 1997 \\
\text { Graminidites } \text { sp. } \\
\text { Haloragacidites sp. } \\
\text { Myrtaceidites verrucosus Partridge, } 1973 \\
\text { Myrtaceidites sp. } \\
\text { Momipites sp. } \\
\text { Nothofagidites americanus Zamaloa, } 1992 \\
\text { Nothofagidites anisoechinatus Menéndez \& Caccavari, } 1975 \\
\text { Nothofagidites dorotensis } \text { Romero, } 1973 \\
\text { Nothofagidites flemingii (Couper) Potonié, } 1960 \\
\text { Nothofagidites menziesii type } \\
\text { Nothofagidites nanus } \text { Romero, 1977 } \\
\text { Nothofagidites rocaensis Romero, } 1973 \\
\text { Nothofagidites saraensis Menéndez \& Caccavari, 1975 } \\
\text { Propylipollis rynthius (Stover y Partridge) Nañez et al., 2009 } \\
\text { Proteacidites subscabratus (Couper) Harris, 1965 } \\
\text { Proteacidites symphyonemoides Cookson, 1950 } \\
\text { Pseudowinterapollis couperi (Krutzsch) Mildenhall, 1979 } \\
\text { Psilamonocolpites grandis (Van der Hammen) Van der Hammen \& García, } 1966 \\
\text { Psilatricolporites salamanquensis Archangelsky \& Zamaloa, 1986 } \\
\text { Restioniidites sp. } \\
\text { Rhoipites baculatus Archangelsky, 1973 } \\
\text { Rhoipites minusculus Archangelsky, 1973 } \\
\text { Rhoipites sp. A (in Quattrocchio, 1980) } \\
\text { cf. Rousea patagonica Archangelsky, 1973 } \\
\text { Spinizonocolpites sp. (Archangelsky, 1973) }\end{array}$ & $\begin{array}{c}\text { Malvaceae } \\
\text { Chenopodiaceae } \\
\text { Onagraceae } \\
\text { Poaceae } \\
\text { Haloragaceae } \\
\text { Myrtaceae } \\
\text { Myrtaceae (cf. Myrceugenia) } \\
\text { Juglandaceae } \\
\text { Nothofagaceae } \\
\text { Nothofagaceae } \\
\text { Nothofagaceae } \\
\text { Nothofagaceae } \\
\text { Nothofagaceae } \\
\text { Nothofagaceae } \\
\text { Nothofagaceae } \\
\text { Nothofagaceae } \\
\text { Proteaceae } \\
\text { Proteaceae } \\
\text { Proteaceae } \\
\text { Winteraceae } \\
\text { Palmae } \\
\text { Restionaceae } \\
\text { Rutaceae-?Araliaceae } \\
\text { Loganiaceae } \\
\text { Rutaceae } \\
\text { Adoxaceae } \\
\text { Palmae } \\
\end{array}$ \\
\hline
\end{tabular}


Table 2. Cont.

\begin{tabular}{|c|c|}
\hline Fossil taxon & Botanical affinity \\
\hline \multicolumn{2}{|l|}{ Angiosperm pollen } \\
\hline $\begin{array}{l}\text { Striatricolporites spp. } \\
\text { Triatriopollenites bertelsii Archangelsky, } 1973 \\
\text { Triorites minor Couper, } 1953\end{array}$ & Myricaceae \\
\hline \multicolumn{2}{|l|}{ Fungi } \\
\hline \multicolumn{2}{|l|}{$\begin{array}{l}\text { Desmidiospora willoughbyi (Bradley, 1987) Ethridge Glass et al., } 1986 \\
\text { cf. Diporicellaesporites navicularis Katgutkar, } 1993 \\
\text { Diporisporites } \mathrm{cf} \text {. psilatus Kumar, } 1990 \\
\text { Fractisporonites } \mathrm{sp} . \\
\text { Hilidicellites } \mathrm{sp} . \\
\text { Hypoxylonites } \mathrm{sp} . \\
\text { Inapertisporites cystoides (Ambwani) Kalgutkar \& Jansonius, } 2000 \\
\text { Inapertisporites elencantense Sepúlveda, } 1980 \\
\text { Monoporites abruptus Sheffy \& Dilcher, } 1971 \\
\text { Monoporisporites lagenarius Song \& Luo, } 1989 \\
\text { Multicellaesporites } \text { sp. } \\
\text { Multicellites } \mathrm{sp} \text {. }\end{array}$} \\
\hline \multicolumn{2}{|l|}{ Organic walled paleomicroplankton } \\
\hline $\begin{array}{l}\text { ?Apteodinium sp. } \\
\text { ?Batiacasphaera baculata Drugg, } 1970 \\
\text { Batiacasphaera micropapillata Stover, } 1977 \\
\text { Batiacasphaera } \text { spp. } \\
\text { ? Cristadinium sp. } \\
\text { Escharisphaeridia psilata Kumar, } 1986 \\
\text { Hystrichostrogylon sp. } \\
\text { Lejeunecysta communis Biffi \& Grignani, } 1983 \\
\text { Lejeunecysta convexa Matzuoka \& Bujak, } 1988 \\
\text { Lejeunecysta fallax (Morgenroth) Biffi \& Grignani, } 1983 \\
\text { Lejeunecysta globosa Biffi \& Grignani, } 1983 \\
\text { Lingulodinium bergmannii (Archangelsky) Quattrocchio \& Sarjeant, } 2003 \\
\text { Lingulodinium hemicystum McMinn, } 1991 \\
\text { cf. Selenopemphix nephroides (Benedek) Benedek \& Sarjeant, } 1981 \\
\text { Spiniferites sp. } \\
\text { Tuberculodinium vancampoae (Rossignol) Wall, } 1967\end{array}$ & $\begin{array}{l}\text { Dinoflagellata } \\
\text { Dinoflagellata } \\
\text { Dinoflagellata } \\
\text { Dinoflagellata } \\
\text { Dinoflagellata } \\
\text { Dinoflagellata } \\
\text { Dinoflagellata } \\
\text { Dinoflagellata } \\
\text { Dinoflagellata } \\
\text { Dinoflagellata } \\
\text { Dinoflagellata } \\
\text { Dinoflagellata } \\
\text { Dinoflagellata } \\
\text { Dinoflagellata } \\
\text { Dinoflagellata } \\
\text { Dinoflagellata }\end{array}$ \\
\hline \multicolumn{2}{|l|}{ Algae } \\
\hline $\begin{array}{l}\text { Botryococcus } \mathrm{sp} . \\
\text { ?Celyphus rallus }\end{array}$ & $\begin{array}{c}\text { Chlorococcales } \\
\text { Rivulariaceae }\end{array}$ \\
\hline \multicolumn{2}{|l|}{ Others } \\
\hline Foraminiferal test linings & \\
\hline
\end{tabular}

The frequent presence of gymnosperm suggests that the parent plants were present in quite large numbers near to the site of deposition. Their anemophilous pollen grains (Podocarpidites spp. and Microcachryidites) characterize the regional input of palynomorphs in the depositional site.

The Araucariaceae community may have occupied ecotones associated with lowlands (García et al., 2006). Some authors related them even with coastal (Abbink, 1998) and/or swampy (Whitaker et al., 1992) communities. The morphologic and structural features of their pollen grains suggest that they are not suitable for transport over large distances or for eolian dispersion. The presence of araucariacean pollen grains in high proportions may be related to forests of altitude or to relatively lower areas where pollen was transported principally by fluvial currents (Martínez et al., 1996). In this paper due to the increase of Araucariaceae associated with a high frequency of pteridophytic spores in the regressive events, a lowlands scenario is suggested.

Phyllocladidites mawsonii Cookson is very similar to pollen of Lagarostrobus franklinii (Hook) Quinn. Today L. franklinii is restricted to high-rainfall areas in western Tasmania, where the annual precipitation is up to $2,500 \mathrm{~mm}$. The habitat is banks of rivers and swampy flats to $750 \mathrm{~m}$.

Most of the Myrtaceae pollen corresponds to Myrtaceidites verrucosus, a rainforest type (Martin, 1997).

The presence of Palmae, even though palms are found in tropical and subropical climates, in Chile reaches the $35^{\circ} 30^{\prime} \mathrm{S}$ surviving $-15^{\circ} \mathrm{C}$ during short periods in winter (Ottone, 2009).

Among the pteridophytas Cyatheacidites annulatus Cookson and Deltoidospora minor Couper and D. australis Couper are recognized. Dispersed spores of Cyatheacidites are found in rocks of Cretaceous and Cenozoic age, and are virtually identical to those of the living monotypic 


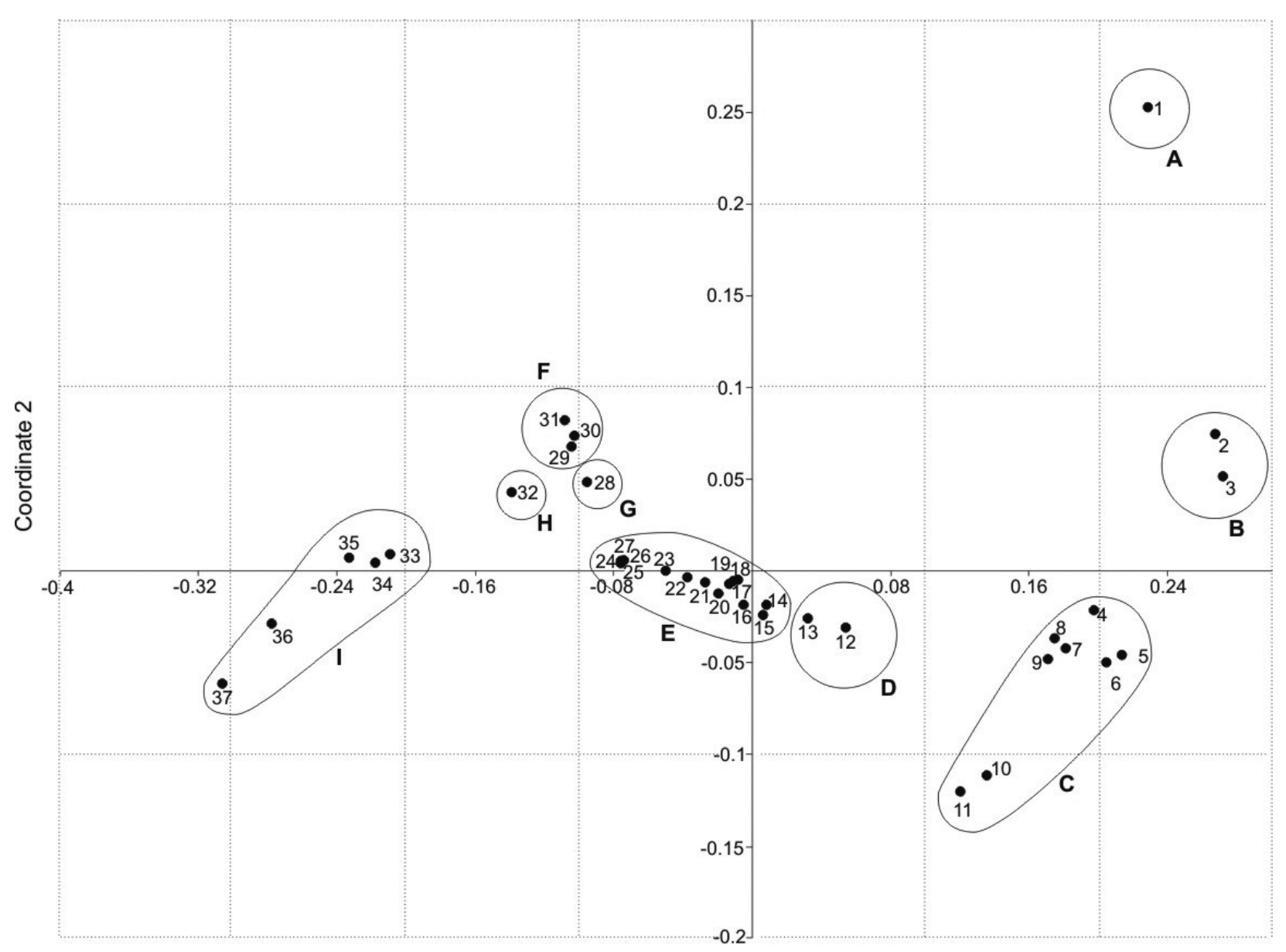

Coordinate 1

Figure 4. Groupings of palynological assemblages based on the non-parametric multidimensional scalin (N-MDS) technique. A-B, Danian, Cerro Bororó and Salamanca formations; C, Eocene, Huitrera and Río Turbio formations; D, late Eocene-?early Oligocene, Sloggett and Troncoso formations; E, Oligocene, Salto del Macho and Río Foyel formations, and "basal muddy section" of the San Julián Formation; F-G, late Oligocene, San Julián (Playa La Mina) and Río Leona formations; H, late Oligocene-early Miocene (Aries X-1 well); I, early-middle Miocene (Monte León and Chenque formations).

fern, Lophosoria according to Kurmann \& Taylor (1987). Oligocene-early Miocene macrofossils of parts of a fertile frond are assigned to the extant South American species Lophosoria quadripinnata (Gmel.) C. Chr. These macrofossils bear the dispersed spore species Cyatheacidites annulatus Cookson ex Potonié (Hill et al., 1999). Living L. quadripinnata is a large fern, now restricted to South America, where it ranges over more than $70^{\circ}$ of latitude and occurs primarily in cloud forest in the tropics and in cool, wet regions further south. It grows in a variety of habitats and readily colonizes disturbed sites such as road cuttings and landslips, and may persist in logged forest, in pastures and in burned areas. Deltoidospora spores were found from Eocene-Oligocene polypodiaceous ferns close to Acrostichum by Collinson (1978). Anzótegui (2006) recognized a palustrine community with rooted herbaceous: Acrostichum cf. A. aureum L. in the middle to upper Miocene of the NW of Argentina. Presence of Pteridaceae, Pteris semiadnata Phil. (Muricingulisporis chenquensis Barreda) was registered. This plant inhabits humid areas, with almost constant rainfall between 5-1370 m.o.s.l. (metres over sea level), in Chile (Rodríguez, 1995).

\section{SEDIMENTARY CYCLES}

The palynological data are discussed in order to reconstruct the paleo-oceanographic and paleoclimatic conditions of the studied section.

The parameter used here for the reconstruction of relative sea-level changes is the relation of terrigenous sporomorphs to marine palynomophs $(\mathrm{t} / \mathrm{m})$. Within a section, the values of the $\mathrm{t} / \mathrm{m}$ relation may fluctuate depending on relative sea level changes, phytoplankton productivity or stronger fluvial/eolic input of terrigenous sporomorphs into the basin. They also depend on episodic regional climatic changes, which produce changes in quantitative (and qualitative) sporomorphs input.

When, for example, a progradational package in a highstand systems tract is considered in detail, it is found to consist of a series of smaller packages of beds, each of which shows an upward shallowing, progradational trend. In the terminology of sequence stratigraphy, this group of beds is called a "parasequence".

In the Río Foyel Formation, homogeneous pelite deposits were identified; the parasequences are identified on the basis of the biofacies characteristics, which indicate upward swallowing. 

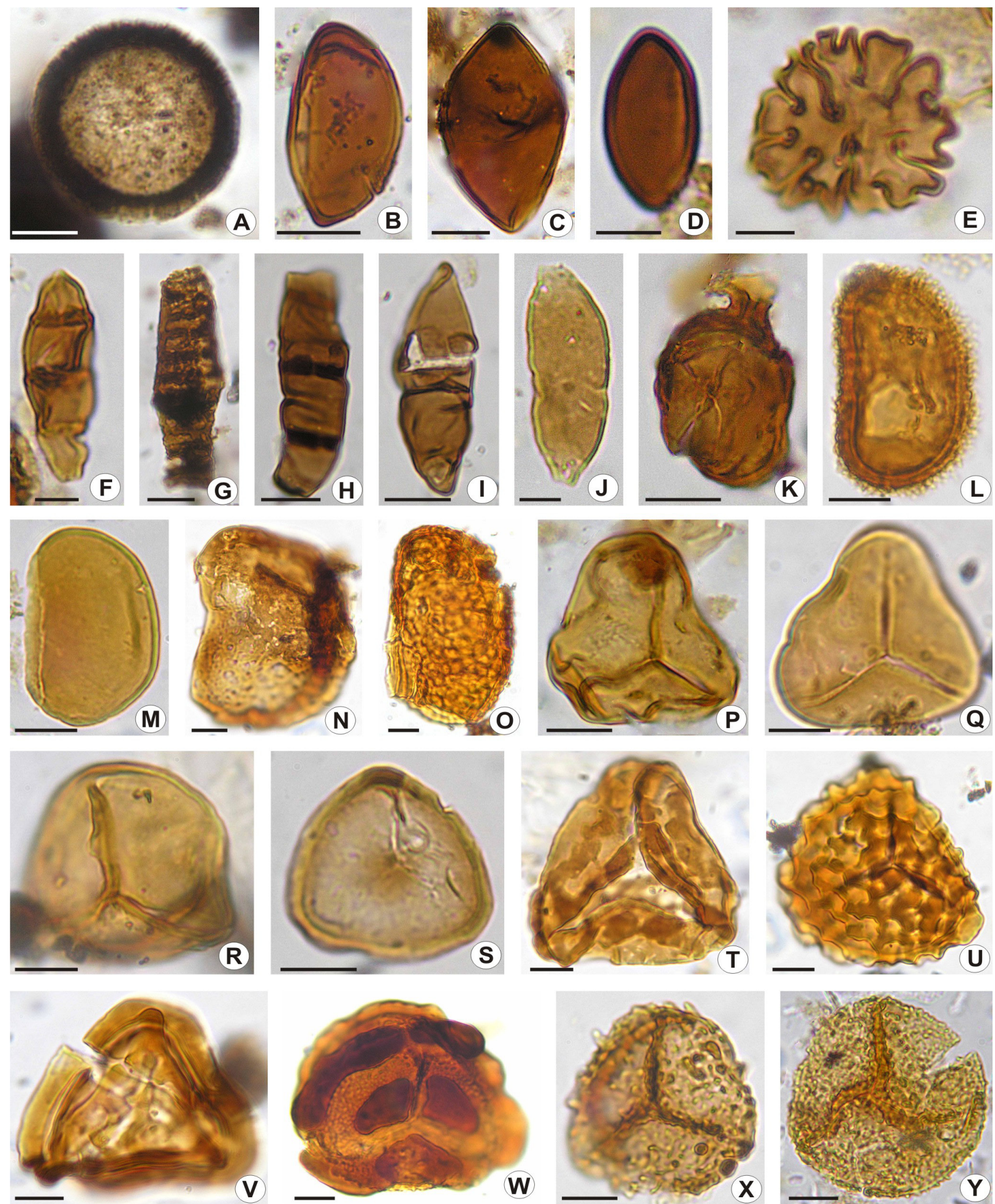

Figure 5. A, Inapertisporites cystoides (Ambwani) Katgutkar \& Jansonius, UNSP RF 2312b: X6. B, Hypoxylonites sp., UNSP RF 2230c: P32. C, Monoporites abruptus Sheffy \& Dilcher, UNSP T 2223b: P66/1. D, Inapertisporites elencantense Sepúlveda, UNSP T 2223b: Z56/4. E, Desmidiospora willoughbyi (Bradley) Ethridge Glass, Brown \& Elsik, UNSP T 2223b: L19/4. F, Multicellaesporites sp., UNSP RF 2300c: T9. G, Fractisporonites sp., UNSP RF 2312b: W5/4. H, Multicellaesporites sp., UNSP SM 2297b: X51/2. I, Hilidicellites sp. UNSP T 2223c: V55. J, Diporisporites cf. D. psilatus Kumar, UNSP RF 2229c: V57. K, Monoporisporites lagenarius Song \& Luo, UNSP RF 2229c: X64/4. L, Peromonolites vellosus Partridge in Stover \& Partridge, UNSP RF 2311: V6. M, Laevigatosporites ovatus Wilson \& Webster, UNSP RF 2228c: X28/3. N, Reticuloidosporites tenellis Krutzsch UNSP SM 2297b: R49. O, Polypodiisporites inangahuensis (Couper) Potonié emend. Pocknall \& Mildenhall, UNSP RF 2311c: F45. P, Cyathidites subtilis Partridge, UNSP RF 2231c: S65/3. Q, Deltoidospora minor (Couper) Pocock, UNSP SM 2297b: K48/4. R, Biretisporites crassilabratus Archangelsky, UNSP SM 2297b: B30/4. S, Cingutriletes australis (Cookson) Archangelsky, UNSP SM 2297b: J54. T, Matonisporites ornamentalis (Cookson) Partridge, in Stover \& Partridge, UNSP SM 2297b: R52/1. U, Ischyosporites areapunctatis (Stuchlik) Barreda, UNSP SM 2297b: V9. V, Muricingulisporis chenquensis Barreda, UNSP SM 2297b: W53/1. W, Cyatheacidites annulatus Cookson, UNSP SM 2297b: V56. X, Baculatisporites turbioensis Archangelsky, UNSP SM 2297b: R58. Y, Baculatisporites sp., UNSP RF 2228c: S44. Scale bars = $10 \mu \mathrm{m}$, except in D, E, F, J = $5 \mu \mathrm{m}$. 

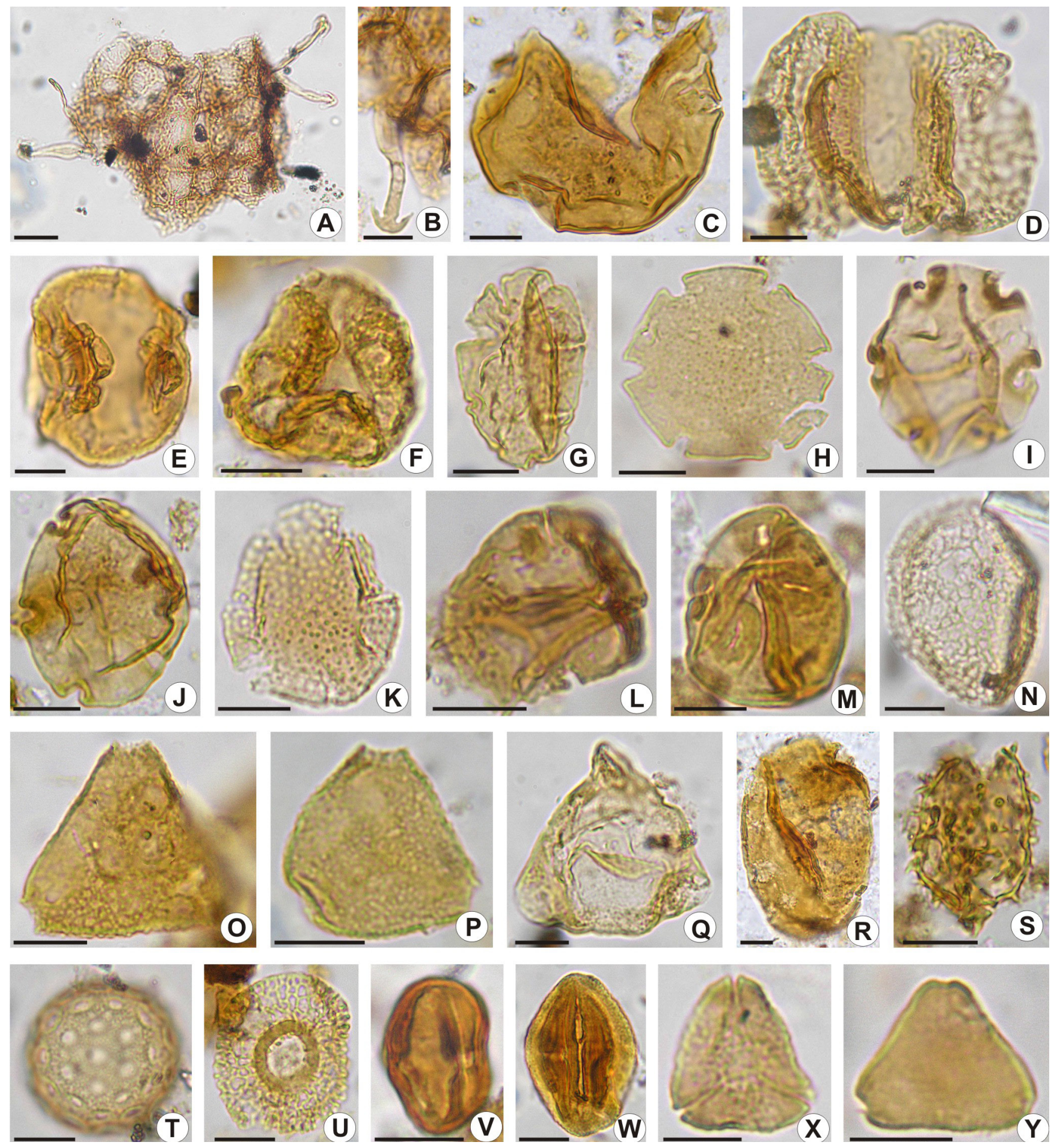

Figure 6. A-B, Azolla sp.: A, UNSP SM 2297: N36/2. B, Detail of Glochidium, UNSP SM 2297b: V20/2. C, Taxodiaceaepollenites hiatus (Potonié) Kremp, UNSP RF 2231c: D17. D, Podocarpidites marwickii, UNSP SM 2297: S11/3. E, Phyllocladidites mawsonii, UNSP SM 2297b: T30/1. F, Trisaccites micosaccatum (Cookson) Couper, UNSP RF 2300c: U9/3. G, Nothofagidites cf. N. dorotensis Romero, UNSP RF 2300c: Y50/1. H, Nothofagidites fuegiensis Menéndez \& Caccavari, UNSP RF 2300d: X52/4. I, Nothofagidites rocaensis Romero, UNSP SM 2297b: Y58/2. J, Nothofagidites saraensis Menéndez \& Caccavari, UNSP RF 2228c: O9/4. K, Nothofagidites "menziesii type" UNSP SM 2297b: N25/2. L, Nothofagidites sp. UNSP RF 2300c: W37/1. M, Haloragacidites sp., UNSP RF 2229c: U13. N, cf. Rousea patagonica Archangelsky, UNSP SM 2297: B29/4. O, Proteacidites symphyonemoides Cookson, UNSP RF 2300c: R12/1. P, Proteacidites sp. UNSP RF 2300d: A32. Q, Corsinipollentites atlantica Barreda, UNSP SM 2297b: R49. R, Psilamonocolpites grandis (Van der Hammen) Van der Hammen \& García, UNSP RF 2233c: O46. S, Spinizonocolpites sp. UNSP RF 2229c: N30. T, Chenopodipollis chenopodiaceoides (Martin) Truswell, UNSP SM 2297b: X23/3. U, Pseudowinterapollis couperi (Krutzsch) Mildenhall, UNSP RF 2300c: B12. V, Rhoipites minusculus Archangelsky, UNSP RF 2234c: V21/1. W, Rhoipites baculatus Archangelsky, UNSP RF 2229c: U42. X, Myrtaceidites verrucosus Partridge, UNSP SM 2297b: S20/2. Y, Triorites minor Couper, UNSP RF 2300d: W44/3. Scale bars $=10 \mu \mathrm{m}$, except in $A=20 \mu \mathrm{m}$. 

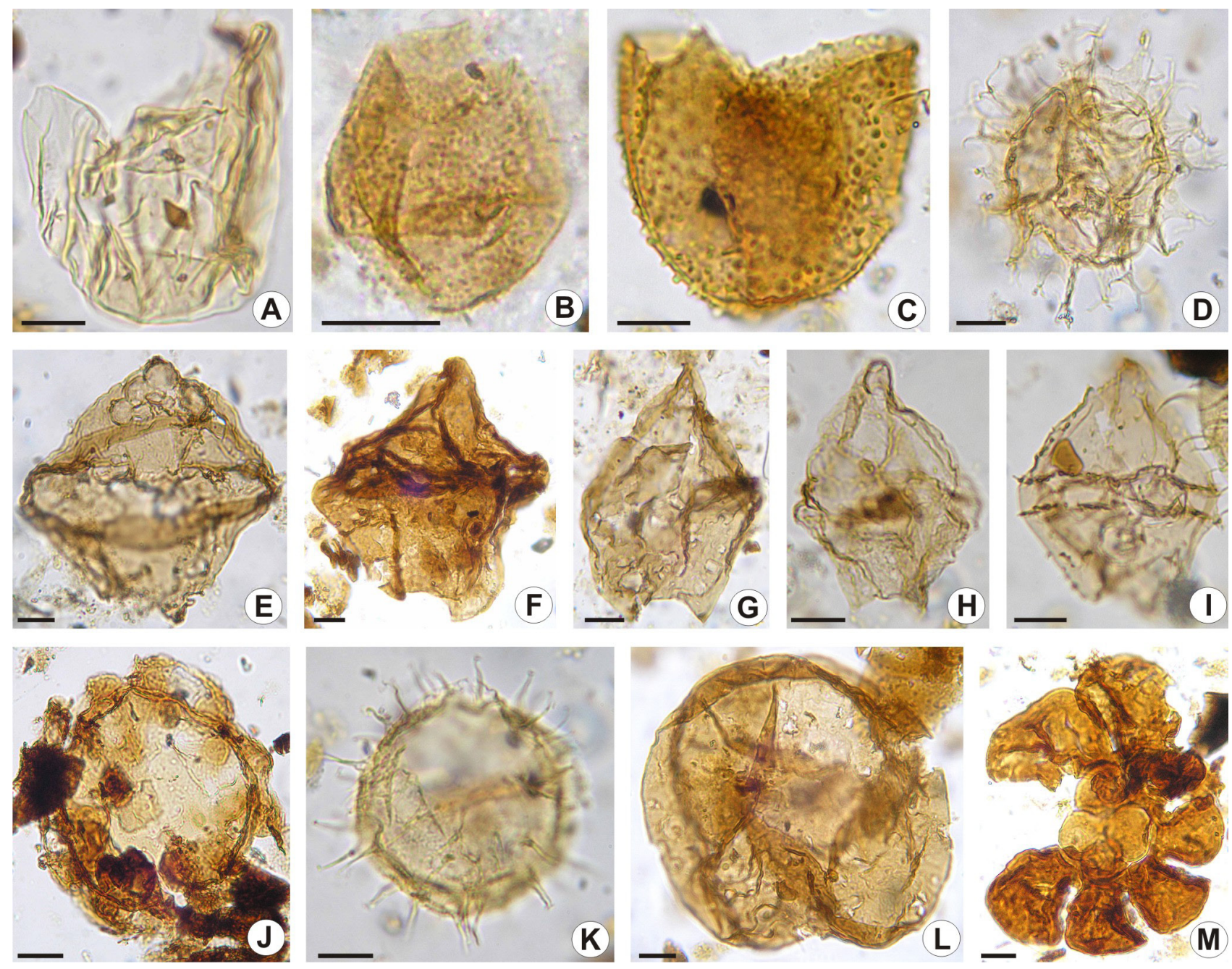

Figure 7. A, Escharisphaeridia psilata Kumar, UNSP T 2223c: T15. B, Batiacasphaera micropapillata Stover, UNSP RF 2233c: Z46. C, ?Batiacasphaera baculata Drugg, UNSP RF 2228c: H24/3. D, Hystrichostrogylon sp., UNSP RF 2300d: O35/2. E, Lejeunecysta globosa Biffi \& Grignani, UNSP RF 2299b: K16/3. F, Lejeunecysta fallax (Morgenroth) Biffi \& Grignani, UNSP RF 2227c: U61/2. G, Lejeunecysta communis Biffi \& Grignani, UNSP RF 2299b: G37/4. H, Lejeunecysta convexa Matsuoka \& Bujak, UNSP RF 2300c: B53. I, ?Cristadinium sp., UNSP RF 2300c: Y30/2. J, Tuberculodinium vancampoae (Rossignol) Wall, UNSP RF 2300c: Z52. K, Lingulodinium bergmannii (Archangelsky) Quattrocchio \& Sarjeant, UNSP RF 2300c: Z26. L, cf. Selenopemphix nephroides (Benedek) Benedek \& Sarjeant, UNSP RF 2227c: K33/3. M, Foraminiferal test linings UNSP RF 2228c:V43/4. Scale bars $=10 \mu \mathrm{m}$

These results are only an approach to this concept, due to limited fertile sampling. The bounding surfaces of parasequences are defined as surfaces of flooding which represent a relative sea level rise (marine flooding surfaces) (Figure 8).

In the studied section, the relation between terrestrial/ marine palynomorphs in Troncoso Formation indicates the general dominance of terrigenous over marine elements. The frequent presences of gymnosperm pollen (46\%) with Araucariaceae $(33.6 \%)$ dominate the spectrum, indicating temperate-humid conditions. The abundance of bryophytic/ pteridophytic spores in general denotes humid local condition, and the pteridophytic polypodiaceous ferns close to Acrostichum (Deltoidospora) reflect palustrine communities.

The dinocysts assemblage corresponds to only proximate cysts (Escharisphaeridia spp.) indicative of near shore conditions. The presence of Algae corresponds to Botryococcus sp. Colonies of Botryococcus are common in littoral areas where they accumulate in quiet and wind-protected sections (Tyson, 1995) with freshwater influence.
In Salto del Macho Formation, there is a retraction of the gymnosperm forest, associated with the dominance of Nothofagus Forest (30\%), indicating temperate-humid condition. The pteridophytic spores $(45 \%)$ correspond in particular to Muricingulisporis chenquensis (Pteridaceae). These plants inhabit humid areas, with almost constant rainfall.

The assemblage of dinocysts corresponds to Escharisphaeridia spp., indicative of a littoral marine environment. Similar conditions are inferred from 13 fertile samples of Salto del Macho Formation, in the same section, by Cornou et al. (2012).

In Río Foyel Formation, in the lower part of the section (from sample F21 to F23), only the "presence" (see Material and Methods) of palynomorph is registered (Pteridophytic, Gymnosperm, Nothofagaceae, Loganiaceae, Myrtaceae and dinocysts). In F23 the presence of Tuberculodinium vancampoae (R. Rossignol) Wall, suggest an estuarine and inner neritic environments, with warm-temperate to warm water temperatures (Wall et al., 1977; Harland, 1983).

Samples F24 to F27, show an upward shallowing sequence, 


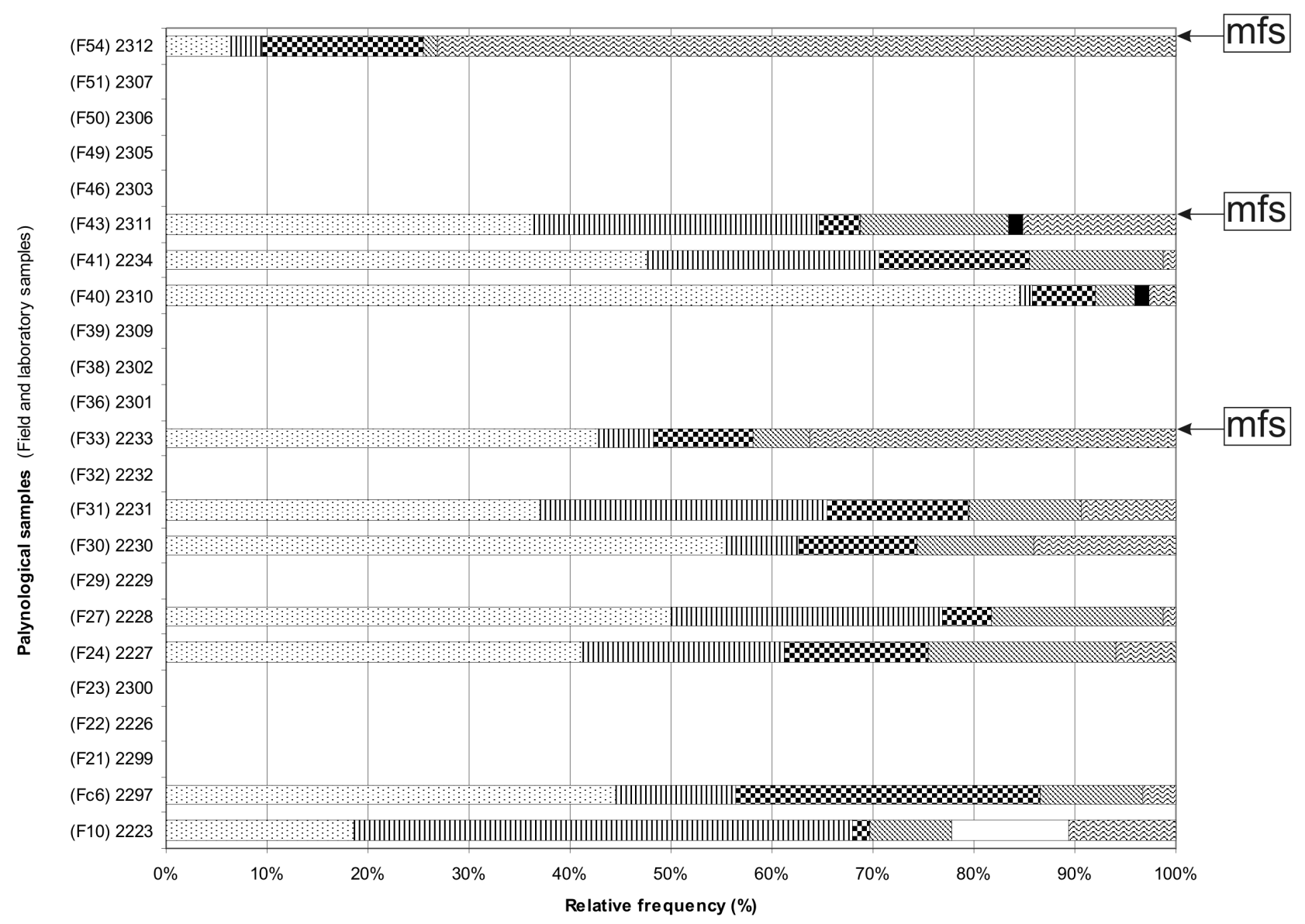

\section{Bryophyta / Pteridophyta III Gymnosperm \\ Nothofagaceae \\ Freshwater algae \\ - Foraminiferal test linings \\ Dinoflagellate + acritarchs \\ $\longleftarrow \mathrm{mfs}$ marine flooding surface}

Other angiosperms

Figure 8. Relation between terrestrial and marine palynomorphs (suprageneric groups). Only stratigraphic levels with quantitative analysis are documented.

indicated by the decrease of dinocysts $(6 \%$ in F 24 to $1.2 \%$ in F27). The pteridophytic dominate the spectrum $(41.2 \%$ in F24 to $50 \%$ en F27). In the regression (F27), the Araucariaceae (6.1\%) income is associated with a decrease of the Nothofagaceae (14.1\% in F24 to $4.8 \%$ in F27) and an increase of the Myrtaceae (3.5\% in F24 to $6.1 \%$ in F27). Phyllocladidites mawsonii Cookson ex Couper have the same representativity (aproximatly $11 \%$ in both samples). This pulse could be associated with temperate to warm-humid climatic condition.

In F30, again, a marine influence is registered (dinocysts $14.1 \%$ with Batiacasphaera spp., Lejeunecysta globosa Biffi \& Grignani and Lingulodinium bergmannii Archangelsky), associated with the income of Palmae (4.6\%), Nothofagaceae (11\%), Myrtaceae (4.6\%) and Poaceae (4.6\%). The pteridophytic spores still dominate the spectrum (55.7\%). Probably, this sample reflects warm conditions.

In sample $\mathrm{F} 31$, there is a relative decrease of marine influence $(9.3 \%$, only Lejeunecysta spp. and Lingulodinium bergmannii are registered). An increase of gymnosperm pollen (28\%) of the montane forest indicates either less humid conditions or the generation of an altitude gradient. Due to a register of humid indicators as Phyllocladidites mawsonii (11.6\%), Nothofagaceae (14\%), and pteridophytic spores $(37 \%)$, the latter could be the most valid explanation.

Probably, marine flooding surface could be detected in F33 where dinocyst reach $36.3 \%$, with ?Batiacasphaera baculata Drugg, B. micropapillata Stover, Lejeunecysta globosa and Lingulodinium bergmannii. There is a register of Pteridophyta (42.9\%), Nothofagaceae $(9.9 \%)$ associated with gymnosperm pollen (5\%), Palmae (2.2\%) and Poaceae (3.3\%). The presence of Palmae suggests relatively warm conditions.

A regressive event (dinocysts: $2.7 \%$ only Lejeunecysta globosa is registered) is present in F40, where the pteridophytic spores $(85.2 \%)$ correspond to pteridophytic polypodiaceous ferns close to Acrostichum (Deltoidospora) and reflect the presence of palustrine environments. The Nothofagaceae (6.3\%), Phyllocladidites mawsonii (1.3\%) and Proteaceae $(6.3 \%)$ indicate humidity. 
A regressive event is also registered at F41 (dinocysts: $2.4 \%$ only Apteodinium sp. and Lingulodinium bergmannii are registered), where the pteridophytic spores reach $47.6 \%$, associated with an increase of the gymnosperm forest $(23 \%)$. Nothofagaceae (14.7\%) and Loganiaceae (3.7\%) are registered.

At F43, a marine flooding surface (dinocysts $15 \%$ with Lingulodinium bergmannii, L. hemicystum McMinn and Spiniferites sp.) could be detected, associated with gymnospermous pollen from the montane forest (28\%).

In F54, the maximum marine flooding surfaces of the studied section are registered (dinocysts: $73.1 \%$, with Lejeunecysta globosa and Lingulodinium bergmannii in high percentages). The Nothofagaceae (15.9\%) have their highest representation associated to the minimum of the pteridophytic spores $(6.4 \%)$ of the profile.

\section{DISCUSSION}

The microfloristic assemblages of El Foyel Group (Troncoso, Salto del Macho and Río Foyel formations), were compared to the following stratigraphic units and localities (Figure 9): Salamanca-Cerro Bororó (Danian,

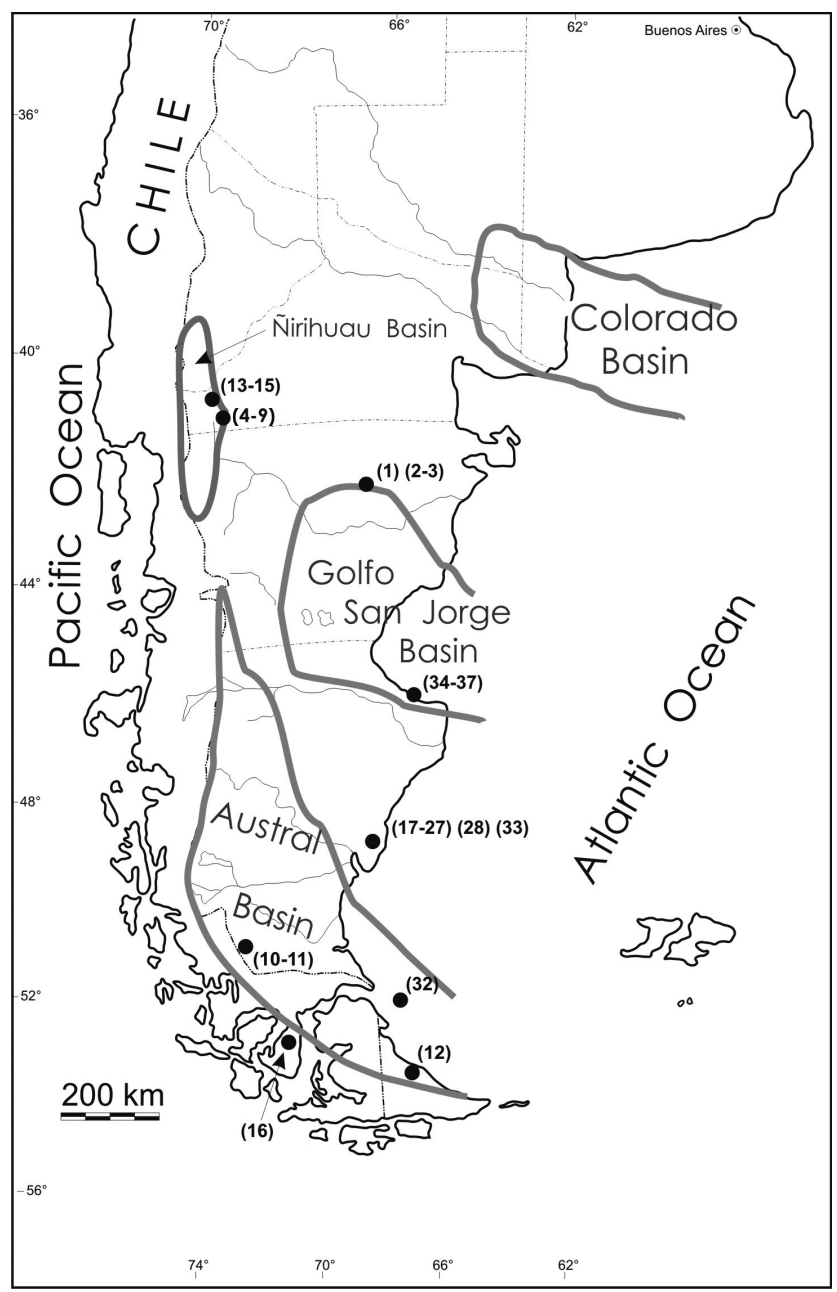

Figure 9. Map of Argentina indicating the sedimentary basins and localities compared in the text. For references of plotted numbers, see Figure 3.
Chubut Province), Huitrera (Eocene, Río Negro and Neuquén provinces), Río Turbio (Eocene, Santa Cruz Province), Sloggett (late Eocene-?early Oligocene, Tierra del Fuego) and Loreto (late Eocene-Oligocene; Magallanes, Chile) formations; El Foyel Group (Troncoso, Salto del Macho and Río Foyel formations, this paper), San Julián (Oligocene, "basal muddy section" and Playa La Mina, Santa Cruz Province); borehole Aries x-1, Tierra del Fuego (late Oligocene-early Miocene); Río Leona (early late Oligocene) and Monte León and Chenque (early-middle Miocene, Chubut Province) formations.

From the analysis of the similarity matrix and of the dendrogram (Figure 3), achieved by application of "cluster analysis", it is evident that the higher values of similarity (close to 1) are between the samples of the Salto del Macho and Río Foyel formations and between Loreto (Fasola, 1969) and San Julián formations in CC3 (Nañez et al., 2009). A second level of similarity is represented between the samples 21, 22 y 23 (FSJ in CC4), associated with the sample 20 (San Julián in CC3).

There is a close similarity between Troncoso and Sloggett formations assigned to the late Eocene-?early Oligocene. The Sloggett Formation is characterized by temperate to temperate-cold climate, very humid and without modern families, such as Poaceae, Onagraceae and Asteraceae (Olivero et al., 1998).

Just as (similarity lower than 0.6) of the Salto del Macho, Troncoso and Río Foyel formations, with the San Julian Formation in Playa La Mina from the late Oligocene (Sample 28), Río Leona Formation (samples 29-31) from the late early Eocene and Aires X-1 (sample 32) from the late Oligoceneearly Miocene.

Chenque and Monte León (Miocene) formations present values lower than 0.4 and, with the formations corresponding to the Eocene (Huitrera and Río Turbio formations) and to the Danian (Salamanca and Cerro Bororó formations), the values are lower than 0.3 .

By means of the N-MDS (Figure 4), it was possible to determine that the samples are mainly grouped according to the analyzed formations. There are six clearly distinct groups, corresponding to the interval Paleocene-Miocene. (A-B) Danian, Cerro Bororó and Salamanca formations; (C), Eocene, Huitrera and Río Turbio formations; (D), late Eocene-?early Oligocene, Sloggett and Troncoso formations; (E), Oligocene, Salto del Macho and Río Foyel formations, and "basal muddy section" of the San Julián Formation; (F-G), late Oligocene, San Julián Formation (Playa La Mina) and Río Leona Formation; (H), late Oligocene - early Miocene (Aries X-1 well); (I), early-middle Miocene (Monte León and Chenque formations).

Then, both the Cluster Analysis and the N-MDS techniques (Figures 3, 4) yielded the same levels of similarity between the samples, which evidences a real difference between the different palynofloras here analyzed.

As in many other paleogene associations of the Patagonia 
(Romero, 1986a, b; Baez et al., 1990; Markgraf et al., 1996), the analyzed formations have podocarpaceae linked to forms which are currently restricted to Tasmania and New Zealand (Phyllocladidites, Dacrycarpites and Microcachryidites), associated to the Nothofagaceae, which are currently part of the Subantartic region. In general, this flora is characterized by Nothofagaceae, Podocarpaceae and, in lower proportion, by Araucariaceae, Myrtaceae and Proteaceae, and they do not present the diversity of angiosperms that appears in the late Oligocene.

In the late Oligocene, a community dominated by Myrtaceae, Palmae and Araucariaceae trees with Podocarpaceae and Nothofagaceae is recognized in San Julián Formation, at Playa La Mina, Santa Cruz Province (Barreda, 1997). However, the presence of small amounts of Anacardiaceae, Malvaceae, Symplocaceae, Ephedraceae, Poaceae, Asteraceae and Chenopodiaceae suggests the development of local open vegetation. The spore-pollen assemblage suggests warm and humid conditions. All of these families are present in the río Foyel section, with the exception of the local open vegetation.

As Barreda et al. (2003) pointed, the almost total absence of herbaceous and shrubby angiosperms in the río Foyel section (upper levels assigned to late Oligocene-early Miocene) is not consistent with the families observed in contemporary formations of the Atlantic coast. Most probably, the reason is that the paleoclimatic conditions allowed a nearly exclusive development of forest on the western side of Patagonia, while near the Atlantic coast some areas with open vegetation began to spread. Pollen assemblages from the Río Foyel Formation (northwestern area) are probably coeval or slightly older (Pöthe de Baldis, 1984; Barreda et al., 2003).

Barreda et al. (2003) provides a detailed discussion on the marine transgression in the Nirihuau Basin. A marine connection with the Pacific Ocean is proposed for the río Foyel section (Malumián et al., 2008; Asensio et al., 2010), due to the presence of crystalline gypsum internal moulds, of both specimens and chambers of Transversigerina cf. $T$. transversa. Palaeoenvironmentally, Transversigerina is a foraminifera genus type of deep water environment, with a tendency towards upper and middle bathyals environments, which is compatible with that of other species present in large numbers in the upper levels of the Río Foyel Formation, such as Sphaeroidina bulloides d'Orbigny. A closed species is T. tenua (Cushman \& Kleinpell), which characterizes the "Patagonian" in the Atlantic coast. In Chile, as opposed to Nirihuau basin, T. transversa is abundant and frequent in Cholchol Formation, Temuco Basin SE of Temuco and Hueyusca Formation, Catamutún Basin, NE of Osorno, ( $c f$. Marchant, 1990). These formations were assigned to the late middle Miocene.

Dinoflagellate assemblages exhibit similarities with those identified in the "Patagonian" deposits outcropping at the southern side of San Jorge Gulf (Barreda \& Palamarczuk, $2000 \mathrm{~b}$ ), but there are no published registers of dinoflagellate coming from the Pacific Ocean at Nirihuau Basin latitude.

\section{CONCLUSIONS}

The Age of El Foyel Group has not been established with precision and generates a lot of controversy. The present statistical results refine the stratigraphic model of Asensio et al. (2005), inferred from stratigraphic relations and absolute data.

In the Río Foyel Formation, homogeneous pelite deposits were registered; this palynological study allows to recognizing parasequences, identifiable on the basis of the biofacies characteristics, which indicate upward shallowing. The bounding surfaces of parasequences are defined as surfaces of flooding which represent a relative sea level rise (marine flooding surfaces) through the profile.

In general, in the Río Foyel Formation the regression events are indicated by the decrease on abundance and diversity of dinocysts and the dominance of pteridophytic spores and the gymnospermous pollen. In general, the increase of the Araucariaceae pollen corresponds to the decrease of the Nothofagaceae pollen.

The marine influence is registered by an increase on the abundance and diversity of dinocysts. In this event the Nothofagaceae, Myrtaceae, Palmae and Poaceae increase in abundance. Marine flooding surface could be detected through the Río Foyel Formation, associated with the dominance of the Nothofagaceae over the gymnospermous pollen; with the exceptions of F43, where the gymnosperm pollen dominate over the Nothofagaceae, probably due to the generation of an altitude gradient.

A comparison of these spore-pollen assemblages with others from Patagonia, using multivariate statistical techniques, yields strong similarities between the Troncoso Formation and the Sloggett Formation (late Eocene-?early Oligocene); and between Salto del Macho and Río Foyel formations with Loreto Formation (late Eocene-Oligocene) and San Julián Formation in cabo Curioso area "basal muddy section" (Oligocene).

\section{ACKNOWLEDGEMENTS}

This research was supported by funds from Agencia Nacional de Promoción Científica y Tecnológica, Consejo Nacional de Investigaciones Científicas y Técnicas (CONICET) and Secretaría de Ciencia y Tecnología (SECyT) of the Universidad Nacional del Sur. In particular, we thank the Administración de Parques Nacionales for permitting access to the outcrops. The authors wish to thank W. Volkheimer as well as C. Jaramillo, M.C. Zamaloa and two anonymous referees for reviewing this manuscript and offering very valuable comments and advice.

\section{REFERENCES}

Abbink, O.A. 1998. Palynological investigations in the Jurassic of the North Sea region. Laboratory of Paleobotany and Palynology, Contributions Series, 8:1-192. 
Anzótegui, L. M. 2006. Paleofloras del Mioceno en Los Valles Calchaquies, Noroeste de Argentina. Facultad de Ciencias Exactas y Naturales y Agrimensura, Universidad Nacional del Nordeste, Ph.D. thesis, $266 \mathrm{p}$.

Archangelsky, S. 1972. Esporas de la Formación Río Turbio (Eoceno). Provincia de Santa Cruz. Revista del Museo de La Plata (Nueva Serie), Paleontología, 6:65-100.

Archangelsky, S. 1973. Palinología del Paleoceno de Chubut. I. Descripciones sistemáticas. Ameghiniana, 10:339-399.

Archangelsky, S. 1976. Palinología del Paleoceno de Chubut. III. Análisis numérico. Ameghiniana, 13:169-184.

Archangelsky, S. \& Romero, E. J. 1974. Polen de gimnospermas (coníferas) del Cretácico superior y Paleoceno de Patagonia. Ameghiniana, 11:217-236.

Archangelsky, S. \& Zamaloa, M.C. 1986. Nuevas descripciones palinológicas de las formaciones Salamanca y Bororó, Paleoceno de Chubut (República Argentina). Ameghiniana, 23:35-46.

Asensio, M.A.; Cornou, M.E.; Malumián, N.; Martínez, M.A. \& Quattrocchio, M.E. 2010. Formación Río Foyel, Oligoceno de Cuenca de Ñirihuau: la transgresión pacífica en la Cordillera Norpatagónica. Revista de la Asociación Geológica Argentina, 66:399-405.

Asensio, M.; Zavala, C. \& Arcuri, M. 2005. Los sedimentos terciarios del Río Foyel, Provincia de Río Negro, Argentina. In: CONGRESO GEOLÓGICO ARGENTINO, 16, 2005. Actas, La Plata, p. 271-276.

Asensio, M.; Zavala, C.A. \& Cazau, L.B. 2008. Análisis tectosedimentario de la Formación Salto del Macho, Cuenca de Ñirihuau. Argentina. In: CONGRESO DE EXPLORACIÓN Y DESARROLLO DE HIDROCARBUROS, 7, 2008. Actas, Mar del Plata, p. 565-574.

Báez, A.M.; Zamaloa, M.C. \& Romero, E. 1990. Nuevos hallazgos de microfloras y anuros paleógenos en el noroeste de Patagonia: implicancias paleoambientales y paleobiogeográficas. Ameghiniana, 27:83-94.

Barreda, V.D. 1996. Bioestratigrafía de polen y esporas de la Formación Chenque, Oligoceno tardío?-Mioceno de las provincias de Chubut y Santa Cruz, Patagonia, Argentina. Ameghiniana, 33:35-96.

Barreda, V. 1997. Palinoestratigrafia de la Formación San Julián en el área de Playa La Mina (Provincia de Santa Cruz), Oligoceno de la Cuenca Austral. Ameghiniana, 34:283-294.

Barreda, V.D. \& Palamarczuk, S. 2000a. Palinomorfos continentales y marinos de la Formación Monte León en su área tipo, provincia de Santa Cruz, Argentina. Ameghiniana, 31:3-12.

Barreda, V.D. \& Palamarczuk, S. 2000b. Estudio palinoestratigráfico del Oligoceno tardío-Mioceno en secciones de la costa patagónica y plataforma continental argentina. In: F.G. Aceñolaza \& R. Herbst (eds.) El Neógeno de Argentina, INSUGEO, Serie Correlación Geológica, 14, p. 103-138.

Barreda, V.D.; García, V.; Quattrocchio, M. \& Volkheimer, W. 2003. Edad y paleoambiente de la Formación Río Foyel, Cuenca Nirihuau, provincia de Río Negro, Argentina. Revista Española de Micropaleontología, 35:229-239.

Barreda, V. D.; Palazzesi, L. \& Marenssi, S. 2009. Palynological record of the Paleogene Río Leona Formation (southernmost South America): stratigraphical and paleoenvironmental implications. Review of Palaeobotany and Palynology, 154:22-33. doi:10.1016/j.revpalbo.2008.11.005

Bertels, A. 1980. Estratigrafía y foraminíferos (Protozoa) bentónicos de la Formación Monte León (Oligoceno) en su área tipo, Provincia de Santa Cruz, República Argentina.
In: CONGRESO ARGENTINO DE PALEONTOLOGÍA Y BIOESTRATIGRAFÍA, 2/CONGRESO LATINOAMERICANO DE PALEONTOLOGÍA, 1, 1978. Actas, Buenos Aires, p. 213-273.

Bertels, A. 1993. Foraminíferos bentónicos (Protozoa) de las "Lutitas de Río Foyel" (Oligoceno medio bajo), Provincia de Río Negro, República Argentina. Revista Española de Micropaleontología, 25:23-58.

Bertels, A. 1994a. Los foraminíferos planctónicos (Protozoa) de las "Lutitas de Río Foyel" (Oligoceno medio bajo), Cuenca Ñirihuau-Ñorquinco-Cushamen, Provincia de Río Negro, Argentina. Ameghiniana, 31:187-194.

Bertels, A. 1994b. Foraminíferos (Protozoa) y paleoambientes de las "Lutitas de Río Foyel" (Oligoceno medio bajo), Cuenca Ñirihuau-Ñorquinco-Cushamen, Provincia de Río Negro, República Argentina. Revista Española de Micropaleontología, 26:99-108.

Berry, E.W. 1928. Tertiary fossil plants from the Argentine Republic. Proceedings of the United States National Museum, 73:1-27.

Cazau, L. 1972. Cuenca de Ñirihuau-Ñorquinco-Cushamen. In: A.F. Leanza (ed.) Geología Regional Argentina, Academia Nacional de Ciencias de Córdoba, p. 427-740.

Cazau, L.; Mancini, D.; Cangini, J. \& Spalletti, L. 1989. Cuenca del Ñirihuau. In: G.A. Chebli \& L.A. Spalletti (eds.) Cuencas Sedimentarias Argentinas, Serie Correlación Geológica, 6, p. 299-318.

Chiesa, J.O. \& Camacho, H.H. 2001. Invertebrados marinos eocenos de la parte inferior de la Formación Río Foyel, provincia de Río Negro, Argentina. Revista Española de Paleontología, 16:299-316.

Collinson, M.E. 1978. Dispersed fern sporangia from the British Tertiary. Annals of Botany, 42:233-250.

Cornou, M.E.; Martínez, M.A.; Quattrocchio, M.E. \& Asensio, M.A. 2012. Estudio palinológico de esporas de la Formación Salto del Macho, Paleógeno de la Cuenca de Ñirihuau, Argentina. Ameghiniana, 49:1-11. doi:10.5710/AMGH.v49i1(322)

Dettmann, M.E. \& Pocknall, D.T. 1990. Nothofagidites Erdtman ex Potonié, 1960; a catalogue of species with notes on the palaeogeographic distributions of Nothofagus Bl. (southern beech). New Zealand Geological Survey, Palaeontological Bulletin, 60:1-79.

Fasola, A. 1969. Estudio palinológico de la Formación Loreto (Terciario medio), provincia de Magallanes, Chile. Ameghiniana, 6:3-49.

García, V.M.; Quattrocchio, M.E.; Zavala, C.A. \& Martínez, M.A. 2006. Palinofacies y paleoclima del Grupo Cuyo (Jurásico medio) en la Sierra de Chacaico, Cuenca Neuquina, Argentina. Revista Española de Micropaleontología, 38:269-288.

Giacosa, H.; Heredia, N.; Césari, O.; Zubia, M.; González, R. \& Faroux, A. 2001. Descripción geológica de la Hoja 4172-IV, San Carlos de Bariloche, Provincias de Río Negro y Neuquén, Buenos Aires, Servicio Geológico Minero Argentino, Instituto de Geología y Recursos Minerales (Boletín 279).

González Bonorino, G. 1944. Descripción geológica y petrográfica de la Hoja 41b "Río Foyel" (Terr. Río Negro). Boletín de la Dirección de Minas, Geología e Hidrología, 56:1-124.

Hammer, Ø.; Harper, D.A.T. \& Ryan, P. D. 2001. PAST: Paleontological Statistics Software Package for Education and Data Analysis. Palaeontologia Electronica, 4:1-9. doi. org/palaeo-electronica.org/2001_1/past/issue1_01.htm

Harland, R. 1983. Distribution maps of recent dinoflagellate cysts from the North Atlantic and adjacent seas. Palaeontology, 26:321-387. 
Head, M.J.; Norris, G. \& Mudie, P.J. 1989. Palynology and dinocyst stratigraphy of the Upper Miocene and lowermost Pliocene, ODP Leg 105, Site 646, Labrador Sea. Proceedings of the Ocean Drilling Program, Scientific Results, 105:423-451.

Hill, R.S.; Macphail, M.K. \& Jordan, G.J. 1999. Tertiary History and Origins of the Flora and Vegetation. In: J.B. Reid; R.S. Hill; M.J. Brown \& M.J. Hovenden (eds.) The Vegetation of Tasmania, Australian Biological Research Study, p. 39-63.

Hünicken, M. 1955. Depósitos Neocretácicos y Terciarios del extreme SSO de Santa Cruz. Revista del Instituto Nacional de Investigaciones de las Ciencias Naturales y Museo Argentino de Ciencias Naturales "Bernardino Rivadavia”, 4:1-161.

Kovach, W.L. 1989. Comparisons of multivariate analytical techniques for use in Pre-Quaternary plant paleoecology. Review of Paleobotany and Palynology, 60:255-282. doi:10.1016/0034-6667(89)90046-8

Kruskal, J.B. \& Wish, M. 1976. Multidimensional scaling. In: J.L. Sullivan \& R.G. Niemi (eds.) Quantitative Applications in the Social Sciences, Sage University Papers, Beverly Hills, p. 7-11.

Kurmann, M.H. \& Taylor, T.N. 1987. Sporoderm ultrastructure of Lophosoria and Cyatheacidites (Filicopsida): systematic and evolutionary implications. Plant Systematic and Evolution, 157:85-94.

Malumián, N.; Asensio, M.; Cornou, E.; Martínez, M. \& Quattrocchio, M.E. 2008. Formación Río Foyel: la transgresión pacífica en la Cordillera Norpatagónica. In: CONGRESO GEOLÓGICO ARGENTINO, 17, 2008. Actas, Jujuy, p. 861-862.

Marchant, M. 1990. Foraminíferos miocénicos de los estratos de Pupunahue (Provinca de Valdivia: $10^{\circ}$ Región): Determinación de la edad probable y Paleoambiente. In: SIMPOSIO SOBRE EL TERCIARIO DE CHILE, 2, 1990. Actas, Concepción, p. 177-188.

Markgraf, V.; Romero, E. \& Villagrán, C. 1996. History and paleoecology of South American Nothofagus forests. In: T. Veblen, R. S. Hill \& J. Read (eds.) The Ecology and Biogeography of Nothofagus forests, Yale University, p. 354-386.

Martin, H.A. 1987. The Cainozoic history of the vegetation and climate of the Lachlan River Region, New South Wales. Proceedings of the Linnean Society of New South Wales, 109:214-257.

Martin, H.A. 1997. The use of Ecological Tolerances for the Reconstruction of Tertiary Paleoclimates. Australian Journal of Botany, 45:475-492.

Martínez, M.A.; García, V.M. \& Quattrocchio, M.E. 1996. Análisis de componentes principales aplicado el estudio palinológico del Jurásico medio de Cuenca Neuquina, Argentina. In: CONGRESO GEOLÓGICO ARGENTINO, 12/CONGRESO DE EXPLORACIÓN DE HIDROCARBUROS, 3, 1996. Actas, Buenos Aires, p. 171-179.

Martínez, M.A.; Ferrer, N. \& Asensio, M. 2008. Primer registro de algas dulceacuícolas del Paleógeno de la Cuenca de Ñirihuau, Argentina: descripciones sistemáticas y análisis palinofacial. Ameghiniana, 45:719-735.

Melendi, D.L; Scafati, L.H. \& Volkheimer, W. 2003. Palynostratigraphy of the Paleogene Huitrera Formation in N-W Patagonia, Argentina. Neues Jahrbuch für Geologie und Paläontologie, Abhandlungen, 228:205-273.

Nañez, C.; Quattrocchio, M.E. \& Ruiz, L. 2009. Palinología y micropaleontología de las formaciones San Julián y Monte León (Oligoceno - Mioceno temprano) en el subsuelo de Cabo Curioso, provincia de Santa Cruz, Argentina. Ameghiniana, 46:669-693.
Olivero, E.B.; Barreda, V.D.; Marenssi, S.A.; Santillana, S.N. \& Martinioni, D.R. 1998. Estratigrafía, sedimentología y palinología de la Formación Sloggett (Paleógeno continental), Tierra del Fuego. Revista de la Asociación Geológica Argentina, 53:504-516.

Ottone, E.G. 2009. La flora cretácica de Cuenca Neuquina. Revista de la Asociación Geológica Argentina, 65:373-386.

Palamarczuk, S. \& Barreda, V.D. 1998. Bioestratigrafía en base a quistes de dinoflagelados de la Formación Chenque (Mioceno), provincia del Chubut, Argentina. Ameghiniana, 35:415-426.

Palamarczuk, S. \& Barreda, V.D. 2000. Palinologia del Paleógeno tardío-Neógeno temprano, pozo Áries x-1, plataforma continental Argentina, Tierra de Fuego. Ameghiniana, 37:221-234.

Pöthe de Baldis, E.D. 1984. Microfloras Cenozoicas. In: CONGRESO GEOLÓGICO ARGENTINO, 10, 1984. Relatorio, San Carlos de Bariloche, p. 393-411.

Rodríguez, R. 1995. Pteridophyta. In: C. Marticorena \& R. Rodríguez (eds.) Flora de Chile, Ediciones Universidad de Concepción, 1:119-309.

Romero, E.J. 1977. Polen de gimnospermas y fagáceas de la Formación Río Turbio (Eoceno), Santa Cruz, Argentina. Buenos Aires, Fundación para la Educación, la Ciencia y la Cultura, $219 \mathrm{p}$.

Romero, E.J. 1986a. Fossil evidence regarding the evolution of Nothofagus Blume. Annals Missouri Botanical Garden, 73:276283.

Romero, E.J. 1986b. Paleogene phytogeography and climatology of South America. Annals Missouri Botanical Garden, 73:449-461.

Romero, J.E. \& Castro, M.T. 1986. Material fúngico y granos de polen de angiospermas de la Formación Río Turbio (Eoceno), provincia de Santa Cruz, República Argentina. Ameghiniana, 23:101-118.

Romero, E.J. \& Dibbern, M.C. 1984. Floras fósiles cenozoicas. In: CONGRESO GEOLÓGICO ARGENTINO, 9, 1984. Relatorio, San Carlos de Bariloche, p. 373-382.

Romero, J.E. \& Zamaloa, M.C. 1985. Polen de angiospermas de la Formación Río Turbio (Eoceno), provincia de Santa Cruz, Argentina. Ameghiniana, 22:43-51.

Roth, S. 1899. Reconocimiento de la región andina de la República Argentina. Apuntes sobre la geología y paleontología de Río Negro y Neuquén. Revista Museo de La Plata, 9:141-197.

Sepúlveda, E. 1980. Estudio palinológico de sedimentitas intercaladas en la "Serie Andesítica Andina", cordón occidental del Futalaufquen, Chubut. Parte I: restos de hongos. Revista de la Asociación Geológica Argentina, 35:248-272.

Tyson, R.V. 1995. Sedimentary organic matter: organic facies and palynofacies. London, Chapman \& Hall, 615 p.

Volkheimer, W. \& Melendi, D. 1976. Palinomorfos como fósiles guías (3ra. Parte). Técnicas de laboratorio palinológico. Revista Minera, Geología y Mineralogía, Sociedad Argentina de Minería y Geología, 34:19-30.

Volkheimer, W.; Scafati, L. \& Melendi, D. L. 2007. Palynology of a Danian warm climatic wetland in central northern Patagonia, Argentina. Revista Española de Micropaleontología, 39:117-134.

von Ihering, H. 1904. Nuevas observaciones sobre moluscos cretáceos y terciarios de Patagonia. Revista del Museo de La Plata, 11:229-243.

von Ihering, H. 1907. Les Mollusques fósiles du Tertiaire et du Crétacé supérieur de Argenine. Anales Museo Nacional de Buenos Aires, 15:1-611.

von Ihering, H. 1914. Catálogo de molluscos cretáceos e terciarios de Argentina de colleção do Autor. Revista Museu Paulista, 1:9-100. 
Wall, D.; Dale, B.; Lohman, G. P. \& Smith, W. K. 1977. The environmental and climatic distribution of dinoflagellate cysts in modern marine sediments from regions in the North and South Atlantic oceans and adjacent seas. Marine Micropaleontology, 2:121-200. doi:10.1016/0377-8398(77)90008-1

Wehrli, L. 1899. Rapport preliminaire sur mon expedition géologique dens la Cordillera Argentina Chilene du $40^{\circ}$ et $41^{\circ}$ latitude sud
(Región du Nahuel Huapí). Revista del Museo de La Plata, 9:221-242.

Whitaker, M. F; Giles, M. R. \& Cannon, S. J. C. 1992. Palynological review of the Brent Group, UK Sector, North Sea. Geological Society of London, Special Publication, 61:169-202.

Received in March, 2011; accepted in February, 2012. 